Pacific Journal of Mathematics

ON THE EMBEDDING OF SUBALGEBRAS CORRESPONDING
TO QUOTIENT ACTIONS IN GROUP-MEASURE FACTORS 


\title{
ON THE EMBEDDING OF SUBALGEBRAS CORRESPONDING TO QUOTIENT ACTIONS IN GROUP-MEASURE FACTORS
}

\author{
JUdith A. PACKer
}

\begin{abstract}
If $X$ and $Y$ are ergodic $G$ spaces, where $G$ is a countable discrete group and $X$ is an extension of $Y$, we study the embedding of the group-measure von Neumann algebra corresponding to $(Y, G)$ into the group-measure von Neumann algebra corresponding to $(X, G)$. Necessary and sufficient conditions for the existence of a normal faithful conditional expectation are established. Under appropriate conditions the normalizer of the subalgebra is determined, and a correspondence between intermediate quotient actions and intermediate von Neumann algebras is established. A relationship between normal extensions with relatively discrete spectrum and crossed dual products of von Neumann algebras by compact second countable groups is determined.
\end{abstract}

Introduction. A study of the relationship between spectral invariants for ergodic actions of countable abelian groups and the corresponding group-measure factors was undertaken by the author in [15], where, under appropriate conditions, the normalizer of a particular maximal abelian subalgebra of the factor corresponding to the trivial quotient action of the group was shown to depend on the pure point spectrum of the original action. In this paper, intended as a sequel to [15], we investigate in further detail certain invariants of quotient actions of ergodic group actions and the embedding of the associated von Neumann algebra. As in [15], spectral invariants play a particularly fruitful role in our study, and in the case where the quotient action is a free action and there is a relatively invariant measure, we use these invariants to deduce the structure of all unitaries normalizing the subfactor corresponding to the quotient action.

The key notion in the construction of the "normalizer" is Zimmer's idea of relative elementary spectrum of an action over a quotient action [27]. Let $G$ be a countable discrete group. If $(X, \mu, G)$ is an ergodic $G$-space and $(Y, \nu, G)$ is a quotient space with quotient map $\varphi: X \rightarrow Y$, such that $\mu$ is relatively $G$-invariant over $\nu$, one says that a one-cocycle $\alpha$ : $Y \times G \rightarrow S^{1}$ is a relative eigenvalue for $(X, \mu, G)$ with relative eigenfunction $f_{\alpha}: X \rightarrow S^{1}$ if

$$
f_{\alpha}(x g)=\alpha(\varphi(x), g) f_{\alpha}(x) \quad \mu \text { a.e., } \quad \forall g \in G .
$$


The $G$-space $(X, \mu, G)$ is said to have relatively elementary spectrum over $(Y, \nu, G)$ if the relative eigenfunctions generate $L^{2}(X)$. Given an arbitrary $G$-space $(X, \mu, G)$ and ergodic quotient $G$-space $(Y, \nu, G)$, there is a natural injection of $F(Y, G)$ into $F(X, G)$, where $F(Y, G)$ and $F(X, G)$ are the von Neumann algebras associated to $(Y, \nu, G)$ and $(X, \mu, G)$ by the group-measure construction. If $f_{\alpha}$ is a relative eigenfunction for $(X, \mu, G)$ over $(Y, \nu, G)$, there is an associated unitary element, $T_{f_{\alpha}}$, of $F(X, G)$ which normalizes $F(Y, G)$. The main theorem of this paper states that under appropriate conditions every unitary element in $F(X, G)$ normalizing $F(Y, G)$ is of such a form, up to multiplication by a unitary element in $F(Y, G)$. Hence one obtains the following

THeOREM 2.2. Let $(Y, \nu, G)$ be a free quotient action of the ergodic $G$-space $(X, \mu, G)$, where $G$ is a countable discrete group, $(X, \mu)$ is a compact Lebesgue space, and the finite measure $\mu$ is relatively $G$-invariant over $\nu$. Then the von Neumann subalgebra of $F(X, G)$ generated by the unitary elements of $F(X, G)$ which normalize $F(Y, G)$ is equal to $F\left(Z_{e}, G\right)$, where $\left(Z_{e}, \tau, G\right)$ is the maximal quotient action of $(X, \mu, G)$ having relatively elementary spectrum over $(Y, \nu, G)$.

Given a quotient action $(Y, \nu, G)$ of $(X, \mu, G)$ satisfying the appropriate conditions, one may apply the theorem repeatedly to attain an ascending chain of subfactors

$$
F(Y, G) \subset F\left(Y_{1}, G\right) \subset F\left(Y_{2}, G\right) \cdots
$$

each one "normal" in the next corresponding to the descending chain of quotient $G$-spaces $(X, \mu, G) \cdots \rightarrow\left(Y_{2}, \nu_{2}, G\right) \rightarrow\left(Y_{1}, \nu_{1}, G\right) \rightarrow(Y, \nu, G)$ each one being the maximal quotient action with relatively elementary spectrum over the next. Continuing this idea by transfinite induction, we deduce the "length" of the subfactor $F(Y, G)$ in $F(X, G)$, and relate this to the generalized elementary spectrum of $(X, \mu, G)$ over $(Y, \nu, G)$.

In [15] we determined the normalizer of a particular maximal abelian subalgebra $S(X, G)$ in $F(X, G)$ (see $\S 1$ for precise details), where $G$ is abelian and preserves the finite measure $\mu$; recall that the normalizer subalgebra was equal to $F\left(Y_{d}, G\right)$ where $\left(Y_{d}, \nu, G\right)$ is the maximal quotient action of $(X, \mu, G)$ having pure point spectrum. If $\left(Y_{d}, \nu, G\right)$ is a free action, we are thus able to determine the length of $S(X, G)$ in $F(X, G)$, by using Theorem 2.2 and applying the above remarks; the length is the countable ordinal $\eta$ of the maximal quotient action of $(X, \mu, G)$ having generalized elementary spectrum. (Recall from [28] that an action is said to have generalized elementary spectrum if it can be built up from the 
trivial action of $G$ on a point by taking extensions with relatively elementary spectrum and inverse limits.)

When $(Y, \nu, G)$ is not a free quotient action of $(X, \mu, G)$, in certain special cases our methods still allow us to determine the normalizer of $F(Y, G)$ in $F(X, G)$. In particular, when $(Y, \nu, G)$ is the trivial action of $G$ on a point and $F(Y, G)$ is therefore equal to $S(X, G)$ we have the following result:

TheOREM 3.3. Let $(X, \mu, G)$ be a free ergodic action of the countable discrete group $G$ on the compact Lebesgue space $(X, \mu)$ which preserves the finite measure $\mu$, and suppose that $(X, \mu, G)$ is weak-mixing, i.e. the associated unitary representation of $G$ on $L^{2}(X) \ominus \mathbf{C}$ has no finite dimensional subrepresentations: Then $S(X, G)$ is its own normalizer in $F(X, G)$.

When $G$ is an abelian group, this theorem provides a new proof of Nielsen's result in ([13], [15]), which does not use the Takesaki equivalence relation.

A major tool in the proof of Theorem 2.5 is the existence of a faithful normal conditional expectation from $F(X, G)$ onto $F(Y, G)$, i.e. a $\sigma$-weakly continuous faithful projection of norm one $E_{Y}^{X}: F(X, G) \rightarrow F(Y, G)$. In this vein we have the following result, which is related to work of Takesaki [20] and Zimmer [29]:

Theorem 1.3. Let $(X, \mu, G)$ be a $G$ space and $(Y, \nu, G)$ an ergodic quotient space, with quotient $G$-map $\varphi, \mu$ and $\nu$ finite measures. Then there exists a faithful normal conditional expectation of $F(X, G)$ onto $F(Y, G)$ if and only if $\mu$ is equivalent to finite measure $\mu^{\prime}, \nu=\varphi_{*} \mu^{\prime}$, with $\mu^{\prime}$ relatively G-invariant over $\nu$.

When $\mu$ is $G$-invariant and $(X, \mu, G)$ is free and ergodic, $F(X, G)$ is a $\mathrm{II}_{1}$ factor. By a result of Umegaki, $F(X, G)$ thus has faithful normal conditional expectations onto all of its von Neumann subalgebras. This allows us to prove a result of related interest about intermediate subalgebras:

Corollary 1.8. Let $(X, \mu, G)$ be a free ergodic $G$-space, and let $(Y, \nu, G)$ be a free quotient action, where $\mu$ (hence $\nu)$ is finite and $G$-invariant. Then every intermediate von Neumann subalgebra between $F(X, G)$ and $F(Y, G)$ is of the form $F(Z, G)$, where $(Z, \tau, G)$ is some quotient action of $(X, \mu, G)$ which is also an extension action of $(Y, \nu, G)$ (hence every intermediate von Neumann subalgebra is a subfactor). 
One of Zimmer's main results in [27] is that for $\mu$ finite and $G$-invariant, the ergodic $G$-space $(X, \mu, G)$ has relatively elementary spectrum over $(Y, \nu, G)$ if and only if there exists a compact second countable abelian group $A$, and a one-cocycle $\alpha: Y \times G \rightarrow A$ such that $(X, \mu, G)$ is essentially isomorphic as an extension to $\left(Y \times{ }_{\alpha} A, \nu \times \nu_{A}, G\right)$. In this situation, recall from [15] that $F(X, G)$ is *-isomorphic in a natural way to the crossed product $F(Y, G) \times_{\hat{\alpha}} \hat{A}$. In [27] and [28], Zimmer also developed the notion of ergodic extensions having relatively discrete spectrum over quotient actions $(Y, \nu, G)$, and showed that these must take on the form $\left(Y \times_{\alpha} K / H, \mu \times v_{K / H} G\right)$, where $K$ is a compact second countable group, $H$ is a closed subgroup, and $\alpha: Y \times G \rightarrow K$ is a one cocycle. In such a situation it is natural to study the embedding of $F(Y, G)$ into $F\left(Y \times_{\alpha} K, G\right)$; in order to mimic the case where $K$ is abelian one must obtain an analogue for the "crossed product by the dual group" construction. Indeed, such a construction for von Neumann algebras already exists in the mathematical literature; we use here the version due to Nakagami ([11], [12]), the so-called "crossed-dual" product. When $(Y, \nu, G)$ is a free ergodic action and $(X, \mu, G)$ is an ergodic extension with $\mu$ finite and relatively $G$-invariant over $\nu$, we are able to show that $F(X, G)$ is a crossed dual product of $F(Y, G)$ by a separable compact group $K$ with appropriate embedding conditions if and only if $(X, \mu, G)$ is essentially isomorphic as an extension to $\left(Y \times{ }_{\alpha} K, \nu \times \nu_{K}, G\right)$ for some 1-cocycle $\alpha$.

Applying these ideas to the case where $F(Y, G)$ is the hyperfinite $\mathrm{II}_{1}$ factor $\mathbf{R}_{0}$, Nakagami-Takesaki duality and results of Zimmer on cocycles with dense range allow us to prove the existence of an outer action $\alpha$ of $K$ on $\mathbf{R}_{0}$ such that $\mathbf{R}_{0} \times{ }_{\alpha} K$ is a factor, for any compact separable group $K$.

The organization of this paper runs as follows. In the first section, after briefly reviewing notation we discuss the existence of a normal faithful conditional expectation from $F(X, G)$ onto $F(Y, G)$ and determine intermediate subalgebras, under appropriate conditions. In $\S 2$ we discuss the normalizer of $F(Y, G)$ in $F(X, G)$ for $(Y, \nu, G)$ a free quotient action of $(X, \mu, G)$ where $\mu$ is relatively $G$-invariant over $\nu$. In $\S 3$ we study the normalizer of $S(X, G)$ in $F(X, G)$ when $(X, \mu, G)$ has continuous spectrum. In the fourth and final section we study the relationship between normal ergodic extensions with relatively discrete spectrum and crossed-dual products of von Neumann algebras by coactions of compact groups.

The results of this paper are for the most part independent of (although complmentary to) the major results in [15]. The general setting and framework is the same, and we refer the reader to $[15, \S 1]$ for any unexplained notation and terminology. 
The research reported here forms a portion of the author's doctoral thesis written at Harvard University. She would like to acknowledge the many important suggestions and ideas of her adviser, Professor G. Mackey, and to thank him for his constant encouragement.

Some of the results of this paper were announced in [14].

1. Conditional expectations onto subalgebras corresponding to quotient actions. Let $(X, \mu, G)$ be a free ergodic $G$-space, where $G$ is a countable discrete group acting on the compact Lebesgue space $(X, \mu)$ so as to leave the finite measure $\mu$ quasi-invariant. By a classic construction due to Murray and von Neumann, one may form a factorial von Neumann algebra on the space $L^{2}\left(X \times G, \mu \times \nu_{G}\right)$ : for $\gamma \in L^{\infty}(X), g_{1} \in G$ and $u \in L^{2}(X \times G)$ define

$$
\begin{gathered}
T_{\gamma}(u)(x, g)=\gamma(x) u(x, g) \\
U_{g_{1}}(u)(x, g)=r_{X}\left(x, g_{1}\right)^{1 / 2} u\left(x g_{1}, g_{1}^{-1} g\right)
\end{gathered}
$$

where $r_{X}(x, g): X \times G \rightarrow \mathbf{R}^{+}$is the Radon-Nikodym derivative associated to the action of $G$ on $(X, \mu)$. We denote by $F(X, G)$ the von Neumann algebra generated by $\left\{T_{\gamma} \mid \gamma \in L^{\infty}(x)\right\}$ and $\left\{U_{g} \mid g \in G\right\}$. It follows from freeness and ergodicity that $F(X, G)$ is a factor. Denote the von Neumann subalgebra of $F(X, G)$ generated by $\left\{T_{\gamma} \mid \gamma \in L^{\infty}(X)\right\}$ by $R(X, G) ; R(X, G)$ is maximal abelian in $f(X, G)$. Let $S(X, G)$ represent the von Neumann subalgebra of $F(X, G)$ generated by $\left\{U_{g} \mid g \in G\right\}$; if $G$ is abelian $S(X, G)$ is maximal abelian in $F(X, G)$. ([1], [15]).

If $(Y, \nu, G)$ is a quotient action of $(X, \mu, G)$ so that there exists a surjective $G$-equivariant Borel map $\varphi: X \rightarrow Y$ with $\varphi_{*}(\mu)=\nu$, then there exists a natural *-monomorphism of $F(Y, G)$ into $F(X, G)$ which we denote by $\varphi^{*}[15]$.

In this section we wish to investigate the conditions under which there exists a faithful normal conditional expectation of $F(X, G)$ onto $\varphi^{*}(F(Y, G))$. When such an expectation exists, and when the quotient action $(Y, \nu, G)$ is also a free action, we are able to show that $R(X, G)$ is the unique maximal abelian subalgebra in $F(X, G)$ containing $\varphi^{*}(R(Y, G))$. This result plays a crucial role in the next section.

If $\mu$ is $G$-invariant measure then $F(X, G)$ will be a $\mathrm{II}_{1}$ factor, and thus will have faithful normal conditional expectations onto all of its subalgebras. This fact will allow us to establish a correspondence between intermediate von Neumann subalgebras of $F(X, G)$ and $F(Y, G)$ and intermediate $G$-spaces of $(X, \mu, G)$ and $(Y, \nu, G)$, where $(Y, \nu, G)$ is a free quotient action of $(X, \mu, G)$. 
Recall that a faithful normal conditional expectation from a von Neumann algebra $\mathscr{M}$ onto a von Neumann subalgebra $\mathscr{N}$ is a $\sigma$-weakly continuous faithful projection $E$ of norm one from $\mathscr{M}$ onto $\mathscr{N}$. We refer the reader to [21], [24] for further details and results. One useful property of such a map $E$ is that $E(a x b)=a E(x) b, \forall a, b \in \mathscr{N}, \forall x \in \mathscr{M}$ [24]. Another important result, due to Umegaki [25], shows that a finite factor has faithful normal conditional expectations onto all of its von Neumann subalgebras. Thus whenever $G$ preserves the finite measure $\mu, F(X, G)$ has this property. When $\mu$ is only quasi-invariant $F(X, G)$ need not be type II. The following definition, due to Zimmer, is very important in the study of conditional expectations onto subalgebras corresponding to quotient actions.

Definition 1.1 [30]. Let $(X, \mu, G)$ be a $G$ space and $(Y, \nu, G)$ be a quotient $G$ space with quotient $G$-map $\varphi$, so that $\varphi_{*} \mu=\nu$, the measure $\nu$ is termed relatively $G$-invariant (over $\nu$ ) if in the decomposition of $\nu$ over the fibers of $\varphi$,

$$
\mu=\int \mu_{y} d \nu
$$

we have $g_{*} \mu_{y}=\mu_{y g}$ for almost all $y$.

Recall that if $(Y, \nu, G)$ is ergodic and $(X, \mu, G)$ is an extension action with $\varphi: X \rightarrow Y$, then there exists a standard compact measure space $(D, m)$, a one-cocycle $\alpha: Y \times G \rightarrow \operatorname{Iso}(D, \tilde{m})$, where $\operatorname{Iso}(D, \tilde{m})$ represents the group of non singular invertible transformations from $(D, m)$ onto itself, given the smallest Borel structure for which $\theta \rightarrow$ $\int f(\theta(d)) h(d) d m$ is Borel for all real valued Borel bounded functions $f$ and $h$ on $D$, and a Borel isomorphism $\Phi: Y \times D \rightarrow X$ with $\Phi_{*} \mu=\nu \times m$, $\Phi((y, d) g)=\Phi\left(\left(y g, \alpha(y, g)^{-1} d\right)\right)=\Phi((y, d)) g$, and $\Pi_{1}=\varphi \circ \Phi$, where $\Pi_{1}$ is projection from $Y \times D$ onto $Y$ ([31], [3]). If $\mu$ is relatively $G$ invariant over $\nu$ then the cocycle $\alpha$ actually takes on its values in Iso $(D, m)$, the group of measure-preserving non-singular invertible transformations of $(D, m)$ onto itself. Therefore the Radon-Nikodym derivative for the action of $G$ on $(X, \mu)$ can be written as

$$
r_{X}(x, g)=r_{Y}(\varphi(x), g)
$$

where $r_{Y}: Y \times G \rightarrow \mathbf{R}^{+}$is the Radon-Nikodym derivative for the action of $G$ on $(Y, \mu)$. Conversely, if $r_{X}$ satisfies (4), then $\mu$ is relatively $G$-invariant over $\nu[30]$. 
EXAmple 1.2. Let $(Y, \nu, G)$ be a $G$-space, and let $\alpha: Y \times G \rightarrow K$ be a cocycle taking on values in the separable compact group $K$. Form the skew product extension $\left(Y \times_{\alpha} K, \nu \times \nu_{K}, G\right)$ where $\nu_{K}$ is normalized Haar measure on $K$. Then $\nu \times \nu_{K}$ is relatively $G$-invariant over $\nu$.

We now note that if $\mu$ is relatively $G$-invariant over $\nu$ then there exists a $G$-invariant faithful normal conditional expectation $E_{Y}^{X}$ from the von Neumann algebra $L^{\infty}(X)$ to $L^{\infty}(Y)$; simply define $P_{Y}^{X}(f)(y)=\int f(x) d \mu_{y}$ (5), and note that

$$
\begin{aligned}
P_{Y}^{X}\left(U^{g} f\right)(y) & =\int f(x g) d \mu_{y}=\int f(x) d\left(g_{*} \mu_{y}\right) \\
& =\int f(x) d \mu_{y g}=P_{Y}^{X}(f)(y g) .
\end{aligned}
$$

Here we define $U^{g}: L^{\infty}(X) \rightarrow L^{\infty}(X)$ by $U^{g} f(x)=f(x g)$. Therefore $P_{Y}^{X}$ is $G$-invariant; it is clear that $P_{Y}^{X}$ is faithful and normal.

Now let $(X, \mu, G)$ be a $G$ space, and $(Y, \nu, G)$ an ergodic quotient action, where $\varphi: X \rightarrow Y$ is the quotient $G$-map. Recall that every element in $F(X, G)$ has a unique Hilbert algebra representation $\sum_{g \in G} T_{\gamma_{g}} U_{g}$, where $\gamma_{g} \in L^{\infty}(X) \forall g \in G$. If $\mu$ is relatively $G$-invariant over $\nu$, one is naturally tempted to define a map $E_{Y}^{X}: F(X, G) \rightarrow \varphi^{*}(F(Y, G))$ by

$$
E_{Y}^{X}\left(\sum_{g \in G} T_{\gamma_{g}} U_{g}\right)=\varphi^{*}\left(\sum_{g \in G} T_{P_{Y}^{X}\left(\gamma_{g}\right)}\right) .
$$

If the range of $E_{Y}^{X}$ actually is in $\varphi^{*}(F(Y, G))$ then it is easy to verify that the properties of the conditional expectation are satisfied. It is necessary to verify, however, that if $\sum_{g \in G} T_{\gamma_{g}} U_{g} \in F(X, G)$, then $\sum_{g \in G} T_{P_{Y}^{X}\left(\gamma_{g}\right)} U_{g}$ is well defined as an element of $F(Y, G)$.

We will prove this fact by proving

THEOREM 1.3. Let $(X, \mu, G)$ be a compact Lebesgue $G$-space, and suppose that $(Y, \nu, G)$ is an ergodic quotient action, with $\varphi: X \rightarrow Y$ and $\varphi_{*} \mu=\nu$. Then there exists a normal faithful conditional expectation $E_{Y}^{X}$ of $F(X, G)$ onto $F(Y, G)$ if and only if $\mu$ is equivalent to a finite measure $\mu^{\prime}$ with $\varphi_{*} \mu^{\prime}=\nu$ and such that $\mu^{\prime}$ is relatively G-invariant over $\nu$.

Proof. $\Leftarrow$ The unitary operator

$$
U: L^{2}\left(X \times G, \mu \times \nu_{G}\right) \rightarrow L^{2}\left(X \times G, \mu^{\prime} \times \nu_{G}\right)
$$

defined by $U(u)(x, g)=\sqrt{f(x)} u(x, g)$ (where $d \mu^{\prime}=f(x) d \mu$ satisfies $U F(X, \mu, G) U^{*}=F\left(X, \mu^{\prime}, G\right), \quad$ and $\left.U \varphi_{\mu}^{*}(F(Y, G)) U^{*}=\varphi_{\mu^{\prime}}^{*}(F(Y, G))\right)$ 
and so without loss of generality assume $\mu$ is relatively $G$-invariant over $\nu$, with $\mu(X)=\nu(Y)=1$.

Then $r_{X}(x, g)=r_{Y}(\varphi(x), g) \mu$ a.e., $\forall g \in G$. Now $L^{2}\left(Y \times G, \nu \times \nu_{G}\right)$ embeds as a closed subspace of $L^{2}\left(X \times G, \mu \times \nu_{G}\right)$ via the map $\varphi$. Let $Q$ : $L^{2}\left(X \times G, \mu \times \nu_{G}\right) \rightarrow L^{2}\left(Y \times G, \nu \times \nu_{G}\right)$ be the corresponding projection. Note that on $L^{1}(X \times G) \cap L^{2}(X \times G) Q$ is given by $Q(u)(y, g)=$ $\int_{X} u(x, g) d \mu_{y}$, for $\mu=\int \mu_{y} d \nu$. To avoid confusion, we let

$$
U_{g_{1}}^{X}: L^{2}(X \times G) \rightarrow L^{2}(X \times G)
$$

be given by

$$
U_{g_{1}}^{X} u(x, g)=r_{Y}\left(\varphi(x), g_{1}\right)^{1 / 2} u\left(x g_{1}, g_{1}^{-1} g\right) \quad g_{1} \in G
$$

and $U_{g_{1}}^{Y}: L^{2}(Y \times G) \rightarrow L^{2}(Y \times G)$ by

$$
U_{g_{1}}^{Y} h(y, g)=r_{Y}\left(y, g_{1}\right)^{1 / 2} h\left(y g_{1}, g_{1}^{-1} g\right) \quad g_{1} \in G .
$$

By definition $U_{g}^{X} \in F(X, G), U_{g}^{Y} \in F(Y, G)$, and $\varphi_{*}\left(U_{g}^{Y}\right)=U_{g}^{X} \forall g \in G$; previously we have denoted both by $U_{g}$. We now claim that we can identify the von Neumann algebra $Q F(X, G) Q$ with $F(Y, G)$; for $Q F(X, G) Q$ acts on $Q L^{2}(X \times G)=L^{2}(Y \times G)$ and $\forall g \in G Q U_{g}^{X} Q=U_{g}^{Y}$ (since $r_{X}(x, g)$ is a lift of $\left.r_{Y}(y, g)\right)$. Also $\forall \gamma \in L^{\infty}(X), u \in L^{1}(Y \times G) \cap$ $L^{2}(Y \times G)$

$$
\begin{aligned}
Q T_{\gamma} Q u(y, g) & =Q(\gamma(x) u(y, g)) \\
& =\int \gamma(x) d \mu_{y} u(y, g)=T_{P_{Y}^{X}(\gamma)} u(y, g) .
\end{aligned}
$$

Since $L^{1}(Y \times G) \cap L^{2}(Y \times G)$ is dense in $L^{2}(Y \times G)$ we obtain $Q T_{\gamma} Q=$ $T_{P_{Y}^{X}(\gamma)}$. But now it is clear that $E(m)=Q(m) Q$ is a $\sigma$-weakly continuous projection of norm one, so that $Q F(X, G) Q=F(Y, G)$. Therefore $E_{Y}^{X}(m)$ $=\varphi^{*}(Q m Q)$ gives the desired projection of norm one from $F(X, G)$ onto $\varphi^{*}(F(Y, G))$ and it is easy to see that if $m$ is represented by $\sum T_{\gamma_{g}} U_{g}^{X}$,

$$
\begin{aligned}
E_{Y}^{X} & =\varphi^{*}(Q m Q)=\varphi^{*}\left(\sum_{g \in G} T_{P_{Y}^{X}\left(\gamma_{g}\right)} U_{g}^{Y}\right) \\
& =\sum_{g \in G} T_{P_{Y}^{X}\left(\gamma_{g}\right) \circ \varphi} U_{g}^{X} .
\end{aligned}
$$

Finally to show that $E_{Y}^{X}$ is faithful, we note that if $x x^{*}=\sum T_{f_{g}} U_{g}$, for $x \in F(X, G)$ then $f_{e} \geq 0, \mu$ a.e., and $f_{e}=0 \mu$ a.e. $\Rightarrow x=0$. Since for positive $f, P_{Y}^{X}(f)(y) \equiv 0 \gamma$ a.e. if and only if $f \equiv 0 \mu$ a.e., we obtain $E_{Y}^{X}\left(x x^{*}\right)=0 \Rightarrow x=0 \Rightarrow x x^{*}=0$; therefore $E_{Y}^{X}$ is faithful.

$\Rightarrow$ Suppose that $E$ is a faithful normal conditional expectation from $F(X, G)$ to $\varphi^{*}(F(Y, G))$. The measure $\nu$ defines a linear functional on 
$\varphi^{*}(F(Y, G))$ by setting $\dot{\psi}_{\nu}\left(\varphi^{*}\left(\sum T_{f_{g}} U_{g}\right)\right)=\int_{Y} f_{e}(y) d \nu$. Denoting the restriction of $\dot{\psi}_{\nu}$ to $\varphi^{*}(F(Y, G))^{+}$by $\psi_{\nu}$, we obtain a finite normal faithful weight on $\varphi^{*}(F(Y, G))$, i.e. $\psi_{\nu}$ maps $\varphi^{*}(F(Y, G))^{+}$to $[0, \infty)$ and satisfies $\psi_{\nu}(x+y)=\psi_{\nu}(x)+\psi_{\nu}(y), \quad x, y \in \varphi^{*}(F(Y, G))^{+}$, and $\psi_{\nu}(\lambda x)=$ $\lambda \psi_{\nu}(x) \lambda \geq 0, \quad x \in \varphi^{*}(F(Y, G))^{+}$, and $\psi_{\nu}(x)=0 \Rightarrow x=0, \quad x \in$ $\varphi^{*}(F(Y, G))^{+}$; by normal it is means that $\dot{\psi}_{\nu}$ is $\sigma$-weakly continuous. (See [6] for details of this construction.) Then $\dot{\psi}_{\nu} \circ E=\dot{\psi}$ defines a normal faithful finite weight on $F(X, G): \psi$ is normal because $\dot{\psi}_{\nu}$ and $E$ are $\sigma$-weakly continuous; it is faithful because $E$ and $\psi_{\nu}$ are faithful, and it is finite because $\psi_{\nu}$ is finite. Examine the restriction of $\dot{\psi}$ to $R(X, G) \cong$ $L^{\infty}(X, \mu)$. Then $\dot{\psi}$ is a $\sigma$-weakly-continuous linear functional on $L^{\infty}(X, \mu)$, and since $L^{\infty}(X, \mu)_{*}=L^{1}(X, \mu)$, there exists a function $f \in L^{1}(X, \mu)$ with $\dot{\psi}\left(T_{\gamma}\right)=\int_{X} f(x) \gamma(x) d \mu$. Since $\psi$ restricted to $R(X, G)^{+}$is finite, faithful and positive it is clear that the function $f$ must be positive and $\mu$ almost nowhere zero. Therefore the measure $\mu^{\prime}$ defined by $d \mu^{\prime}=f(x) d \mu$ is a positive measure which is equivalent to $\mu$. It is clear that $\nu=\varphi_{*} \mu^{\prime}$ since for every $h(y) \in L^{\infty}(Y, \nu)$

$$
\int_{Y} h(y) d \nu=\psi_{\nu}\left(\varphi^{*}\left(T_{h}\right)\right)=\psi_{\nu}\left(E\left(\varphi^{*}\left(T_{h}\right)\right)\right)=\int_{X} h \circ \varphi(x) d \mu^{\prime} .
$$

Finally, let $P_{e}: L^{2}\left(Y \times G, \nu \times \nu_{G}\right) \rightarrow L^{2}(Y, \nu)$ be projection onto

$$
L^{2}\left(Y \times\{e\}, \nu \times \nu_{e}\right)=L^{2}(Y, \nu) .
$$

Then $P_{e} F(Y, G) P_{e}$ can be identified with a normal projection of $F(Y, G)$ onto $R(Y, G) \cong L^{\infty}(Y, \nu)$ (see [22, p. 364]), and an easy calculation shows that for every $m \in F(X, G) \psi(m)=\psi_{\nu}(E(m))=\psi_{\nu}\left(P_{e} E(m) P_{e}\right)$. In particular if $m=T_{\gamma} \in R(X, G), P_{e} E(m) P_{e} \in L^{\infty}(Y)$ and

$$
\begin{aligned}
\psi_{\nu}\left(P_{e} E(m) P_{e}\right) & =\psi(m)=\int \gamma(x) \mu^{\prime}=\int_{Y}\left(\int_{X} \gamma(x) d \mu_{y}^{\prime}\right) d \nu \\
& =\psi_{\nu}\left(T_{J_{X} \gamma(x) d \mu_{y}^{\prime}}\right),
\end{aligned}
$$

where $\int \mu_{y}^{\prime} d \nu=\mu^{\prime}$ is the decomposition of $\mu^{\prime}$ over the fibers of $\varphi$. By uniqueness of decomposition, if $P_{e} E\left(T_{\gamma}\right) P_{e}=T_{h}$ for $h \in L^{\infty}(Y)$, we must have $h(y)=\int_{X} \gamma(x) d \mu_{y}^{\prime} \nu$ a.e. Then $\forall \gamma \in L^{\infty}(X)$ and $\forall g \in G$,

$$
\begin{aligned}
T_{\int_{X} \gamma(x) d g_{*} \mu_{y}^{\prime}} & =T_{\int \gamma(x g) d \mu_{y}^{\prime}}=\left(P_{e} E\left(U_{g}^{X} T_{\gamma} U_{g}^{X_{1}}\right) P_{e}\right) \\
& =P_{e} U_{g}^{Y} E\left(T_{\gamma}\right) U_{g}^{Y} P_{e}=T_{h(y g)} .
\end{aligned}
$$

Therefore $\int_{X} \gamma(x) d\left(g_{*} \mu_{y}^{\prime}\right)=h(y g)=\int_{X} \gamma(x) d \mu_{y g}^{\prime} \nu$ a.e., so that $g_{*} \mu_{y}^{\prime}=\mu_{y g}^{\prime} \nu$ a.e., by the uniqueness of decomposition. Hence $\mu^{\prime}$ is relatively $G$-invariant over $\nu$, as desired. 
REMARK 1.4. We note that if $\mu$ is relatively $G$ invariant over $\nu$, then the modular automorphism group corresponding to the weight $\psi_{\mu}$ leaves $\varphi_{*}(F(Y, G))$ invariant. Hence direction $\Leftarrow$ of the theorem also follows from applying the main theorem of [21].

REMARK 1.5. In [31] Zimmer proved that for $(X, \mu, G)$ an ergodic extension of $(Y, \nu, G)$, there is a norm one projection from $F(X, G)$ onto $\varphi^{*}(F(Y, G))$ if and only if $(X, Y)$ is an amenable pair of $G$-spaces, which is a more general situation than $\mu$ being relatively $G$-invariant over $\nu$. For our purposes, however, faithfulness and normality are essential, and the projection constructed in [29] need not be either.

The existence of a normal faithful conditional expectation allows us to prove the following lemma, which will play an essential role in the following section.

LEMMA 1.6. Let $(X, \mu, G)$ be an extension of the free ergodic action $(Y, \nu, G)$, with quotient map $\varphi$, and suppose that there exists a faithful normal conditional expectation from $F(X, G)$ onto $\varphi^{*}(F(Y, G))$. Then $\varphi^{*}(R(Y, G))^{\prime} \cap F(X, G)=R(X, G)$.

Proof. Since $F(Y, G)$ and $R(Y, G)$ inject naturally into $F(X, G)$ via the map $\varphi_{*}$, we may identify elements in $F(Y, G)$ with their image in $F(X, G)$. Suppose that $K=\sum_{g \in G} T_{f_{g}} U_{g} \in R(Y, G)^{\prime} \cap F(X, G)$. Then for every $g \in G$, the element $T_{\left(f_{g}\right)} U_{g}$ also commutes with everything in $R(Y, G)$ as well. Let $E_{Y}^{X}$ be the faithful normal conditional expectation from $F(X, G)$ onto $F(Y, G)$. Then $\forall b \in R(Y, G)$,

$$
b E_{Y}^{X}\left(T_{\left|f_{g}\right|}\right)=E_{Y}^{X}\left(b T_{\left|f_{g}\right|}\right)=E_{Y}^{X}\left(T_{\left|f_{g}\right|} b\right)=E_{Y}^{X}\left(T_{\left|f_{g}\right|}\right) b .
$$

Since $R(Y, G)$ is maximal abelian in $F(Y, G)$, we see that $E_{Y}^{X}\left(T_{\left|f_{g}\right|}\right)=T_{\gamma_{g}}$ for some $\gamma_{g} \in L^{\infty}(Y)$. By using the same argument we see that $\forall b \in$ $R(Y, G)$,

$$
\begin{aligned}
b T_{\gamma_{g}} U_{g} & =b\left(E_{Y}^{X}\left(T_{\left|f_{g}\right|}\right) U_{g}\right)=E_{Y}^{X}\left(b T_{\left|f_{g}\right|} U_{g}\right)=E_{Y}^{X}\left(T_{\left|f_{g}\right|} U_{g} b\right) \\
& =E_{Y}^{X}\left(T_{\left|f_{g}\right|}\right) U_{g} b=T_{\gamma_{g}} U_{g} b .
\end{aligned}
$$

Therefore if $g \neq e$, we must have $\gamma_{g} \equiv 0 \nu$ a.e. Since $E_{Y}^{X}$ is faithful and $T_{\left|f_{g}\right|} \in F(X, G)^{+} \cup\{0\}$, we see that $\left|f_{g}\right| \equiv 0 \mu$ a.e. Hence any $K \in$ $R(Y, G)^{\prime} \cap F(X, G)$ must be of the form $T_{f_{e}} U_{e} \in R(X, G)$. Since $R(X, G)$ is clearly contained in $R(Y, G)^{\prime} \cap F(X, G)$, we are done. 
Taken together, Theorem 1.3 and Lemma 1.6 imply that if $(Y, \nu, G)$ is a free ergodic quotient action of $(X, \mu, G)$, and if $\mu$ is relatively $G$-invariant over $\nu$, then $R(Y, G)$ extends to a unique maximal abelian subalgebra of $F(X, G)$, namely $R(X, G)$.

Another rather easy consequence of Theorem 1.3 and Lemma 1.6 is

THEOREM 1.7. Let $(X, \mu, G)$ be an ergodic extension of the free ergodic action $(Y, \nu, G)$, and suppose that $\mu$ is relatively $G$-invariant over $\nu$. Then every von Neumann subalgebra $\mathscr{N}$ such that $F(Y, G) \subset \mathscr{N} \subset(X, G)$ which is also the image of a normal faithful conditional expectation on $F(X, G)$ must be of the form $F(Z, G)$ where $(Z, \tau, G)$ is a quotient action of $(X, \mu, G)$ and an extension action of $(Y, \nu, G)$.

Proof. Let $E_{\mathcal{N}}^{X}$ and $E_{Y}^{X}$ as in Theorem 1.3 be the normal faithful conditional expectations from $F(X, G)$ onto the subalgebras $\mathscr{N}$ and $F(Y, G)$ respectively. Then $E_{Y}^{\mathcal{N}}=E_{Y}^{X}$ restricted to $\mathscr{N}$ defines a normal faithful conditional expectation from $\mathscr{N}$ onto $F(Y, G)$ and it is a trivial verification that $E=E_{Y}^{\mathcal{N}} \circ E_{\mathcal{N}}^{X}$ defines a normal faithful conditional expectation from $F(X, G)$ onto $F(Y, G)$. (In fact $E=E_{Y}^{X}$ although we do not need this.) We now examine the subalgebra $E_{\mathscr{N}}^{X}(R(X, G))=R_{\mathscr{N}}$. For every $n \in R(Y, G)$ and every $\mathscr{Q} \in R(X, G), \quad m E_{\mathscr{N}}^{X}(\mathscr{Q})=E_{\mathscr{N}}^{X}(m \mathscr{Q})=$ $E_{\mathscr{N}}^{X}(2 m)=E_{\mathscr{N}}^{X}(\mathscr{Q}) m$. Therefore $E_{\mathscr{N}}^{X}(\mathscr{Q})$ commutes with everything in $R(Y, G)$. So by applying Lemma 1.6 we see that $R_{\mathscr{N}} \subset R(X, G)$, and furthermore $R_{\mathscr{N}}$ is $G$-invariant. Indeed $\forall g \in G$

$$
U_{g} R(X, G) U_{g^{-1}}=R(X, G)
$$

so that

$$
\begin{aligned}
U_{g} R_{\mathscr{N}} U_{g^{-1}} & =U_{g} E_{\mathscr{N}}^{X}(R(X, G)) U_{g^{-1}}=E_{\mathscr{N}}^{X}\left(U_{g} R(X, G) U_{g^{-1}}\right) \\
& =E_{\mathscr{N}}^{X}(R(X, G))=R_{\mathscr{N}} .
\end{aligned}
$$

Recalling that the action of $\operatorname{Ad} U_{g}$ on $R(X, G)$ corresponds to the action of $G$ on $L^{\infty}(X)$, we see that $R_{\mathscr{N}}$ can be naturally identified with a closed *-subalgebra of $L^{\infty}(X, \mu)$ which is $G$-invariant. Since $R_{\mathscr{N}}$ contains the subalgebra $R(Y, G) \cong L^{\infty}(Y)$ which is itself a $G$-invariant closed *-subalgebra, we may apply [27, Cor. 2.2] twice to show that $R_{\mathscr{N}}$ corresponds to $L^{\infty}(Z, \tau)$ for some quotient action $(Z, \tau, G)$ of $(X, \mu, G)$ which is itself an extension action of $(Y, \nu, G)$. Therefore $\mathscr{N}$ contains the von Neumann subalgebra $F(Z, G)$. Now for any $m \in F(X, G)$ there exists a net $\left\{\sum_{g \in G} T_{f_{g}^{a}} U_{g} \mid \alpha \in A\right\}$ for some index set $A$, converging to $m$ in the weak operator topology, where for fixed $\alpha$ all but a finite number of the $f_{g}^{\alpha}$ are 
identically zero. Since $E_{\mathscr{N}}^{X}$ is normal, the net $\left\{E_{\mathscr{N}}^{X}\left(\sum_{g \in G} T_{f_{g}^{\alpha}} U_{g}\right) \mid \alpha \in A\right\}$ will converge to $E_{\mathscr{N}}^{X}(m)$ in the weak operator topology It is clear that for each $\alpha \in A, E_{\mathscr{N}}^{X}\left(\sum_{g \in G} T_{f_{g}^{\alpha}} U_{g}\right)=\sum_{g \in G} E_{\mathscr{N}}^{X}\left(T_{f_{g}^{\alpha}}\right) U_{g}$ and thus is an element of $F(Z, G)$. Therefore $E_{\mathscr{N}}^{X}(m)$ is an element of $F(Z, G)$.

Hence $\mathscr{N}=E_{\mathscr{N}}^{X}(F(X, G))=F(Z, G)$, as desired.

Theorem 1.7 has the following immediate

COROllary 1.8. Let $(X, \mu, G)$ be an ergodic $G$-space, where $\mu$ is $G$-invariant, and suppose that $(Y, \nu, G)$ is a free quotient action. Then every von Neumann subalgebra $\mathscr{N}$ such that $F(Y, G) \subset \mathscr{N} \subset F(X, G)$ is of the form $F(Z, G)$ for some quotient action $(Z, \tau, G)$ of $(X, \mu, G)$ which is also an extension action of $(Y, \nu, G)$ (and thus $\mathcal{N}$ must be a subfactor $)$.

Proof. Since $\mu$ is finite and $G$ invariant, and $(X, \mu, G)$ inherits freeness from $(Y, \nu, G), F(X, G)$ is a factor of type $\mathrm{II}_{1}$. It follows that $F(X, G)$ has a faithful normal conditional expectation onto any subalgebra. The theorem shows that any intermediate subalgebra $\mathscr{N}$ takes on the desired form. Since $(Z, \tau, G)$ inherits ergodicity from $(X, \mu, G)$ and freeness from $(Y, \nu, G), \mathscr{N}$ will be a factor.

REMARK 1.9. Under the same hypotheses as in the corollary, the following intuitive remarks can be made. The virtual subgroup of $G$ (see [9]) corresponding to the groupoid $Y \times G$ can be considered as "containing" the virtual subgroup of $G$ corresponding to the groupoid $X \times G$. Denote these subgroups by $\mathscr{V}_{Y}$ and $\mathscr{V}_{X}$ respectively. Recall that $F(X, G)$ can be thought of as the von Neumann algebra generated by the left regular representation of the groupoid $X \times G \approx$ " $G / \mathscr{V}_{X}$ " and $F(Y, G)$ can be thought of as the von Neumann algebra generated by the left regular representation of the groupoid $Y \times G \approx " G / \mathscr{V}_{Y}$ ". Then Corollary 1.8 states that the intermediate subalgebras between these two von Neumann algebras correspond exactly to the virtual subgroups lying in between $\mathscr{V}_{Y}$ and $\mathscr{V}_{X}$.

2. The normalizer of subfactors corresponding to quotient actions in $F(X, G)$. One of the main results of [15] was the construction of the unitaries in $F(X, G)$ which normalize $S(X, G)$, where $(X, \mu, G)$ is a free ergodic $g$-space with $G$ countable abelian and measure preserving; these unitaries were completely determined by the pure point spectrum of the group action. Taking $Y=$ pt., the trivial $G$-map $\varphi: X \rightarrow$ pt. gives rise to the injection $\varphi^{*}: F(Y, G) \rightarrow F(X, G)$ with $\varphi^{*}(F(Y, G))=S(X, G)$. Given 
this observation it is natural to attempt to calculate the unitaries in $F(X, G)$ which normalize $\varphi^{*}(F(Y, G))$, where $(Y, \nu, G)$ is a non-trivial quotient action of $(X, \mu, G)$ with quotient $G$-map $\varphi:(X, \mu) \rightarrow(Y, \nu)$. For the case where $\mu$ is relatively $G$-invariant over $\nu$ and the quotient action $(Y, \nu, G)$ is free, we have been able to accomplish this for an arbitrary countable discrete group $G$. The unitaries in $F(X, G)$ which normalize $F(Y, G)$ bear a relationship to Zimmer's action of relatively elementary spectrum, so we briefly review this notion. Since the related concept of relatively discrete spectrum will be needed in Section 4, we discuss the two notions together.

Let $(X, \mu, G)$ be a compact Lebesgue $G$-space and $(Y, \nu, G)$ a quotient ergodic $G$-space, with $\varphi: X \rightarrow Y$ the quotient $G$-map. Suppose that $\mu$ is relatively $G$-invariant over $\nu$, so that in the decomposition of $\mu$ over the fibers of $\varphi \mu=\int \mu_{y} d \nu, g_{*} \mu_{y}=\mu_{y g} \nu$ a.e., $\forall g \in G$.

We may decompose $L^{2}(X, \mu)$ as the direct integral $\int_{Y}^{\oplus} \mathscr{H}_{y} d \nu$, where $\mathscr{H}_{y}=L^{2}\left(\varphi^{-1}(y), \mu_{y}\right)$. For almost every $y \in Y$ and every $g \in G$, there exists a unitary mapping

$$
\alpha(y, g): \mathscr{H}_{y g} \mapsto \mathscr{H}_{y}
$$

given by

$$
\alpha(y, g) f(x)=f(x g) .
$$

It is clear that $\alpha$ satisfies the cocycle identity

$$
\alpha\left(y, g_{1} g_{2}\right)=\alpha\left(y, g_{1}\right) \alpha\left(y g_{1}, g_{2}\right) \text {. }
$$

The map $\alpha$ is an example of a unitary bundle cocycle representation; the reader is referred to [27] for further details on the theory of these objects. We recall from [27] that $(X, \mu, G)$ is said to have relatively elementary (respectively relatively discrete) spectrum over $(Y, \nu, G)$ if the bundle cocycle representation $\alpha$ given above decomposes as the direct sum of one-dimensional (respectively finite dimensional) subbundle representations.

For every ergodic extension $(X, \mu, G)$ of an ergodic action $(Y, \nu, G)$ where $\mu$ is relatively $G$-invariant over $\nu$, there exist maximal quotient actions of $(X, \mu, G)$ having relatively discrete and relatively elementary spectrum over $(Y, \nu, G)$. Denote these extensions by $\left(Z_{d}, \tau_{d}, G\right)$ and $\left(Z_{e}, \tau_{e}, G\right)$ respectively. One of the main results of Zimmer's theory ([27], which can be extended to the case $\mu$ quasi-invariant by using results of Fabec [4]) is that there exists a second countable compact group $K$, and closed subgroups $D \subset E \triangleright K$, such that $K / E$ is abelian, and a cocycle $\beta$ : $Y \times G \rightarrow K$ such that

$$
\left(Z_{e}, \tau_{e}, G\right) \cong\left(Y \times_{\beta} K / E, \nu \times \nu_{K / E}, G\right)
$$


and

$$
\left(Z_{d}, \tau_{d}, G\right) \cong\left(Y \times_{\beta} K / D, \nu \times \nu_{K / D}, G\right) .
$$

(Here by $\cong$ we mean essential isomorphism as extensions.) In particular, $\left(Z_{e}, \tau_{e}, G\right)$ is given by a skew-product $\left(Y \times_{\hat{\beta}} A, \nu \times \nu_{A}, G\right)$ where $A$ is a second countable compact group.

Associated to every one-dimensional subbundle representation of the natural cocycle representation $\alpha$ for an ergodic extension $(X, \mu, G)$ of $(Y, \nu, G)$ there is an $L^{2}$ Borel function $f_{\beta}: X \rightarrow \mathrm{C}$ and a one cocycle $\beta$ : $Y \times G \rightarrow S^{1}$ such that

$$
f_{\beta}(x g)=\beta(\varphi(x), g) f_{\beta}(x) \quad \mu \text { a.e. } \quad \forall g \in G .
$$

Ergodicity then implies that $\left|f_{\beta}\right|$ is constant a.e. and without loss of generality we assume $\left|f_{\beta}\right|=1$.

Definition 2.1. Let $f_{\beta}: X \rightarrow S^{1}$ be a Borel function and $\beta: Y \times G \rightarrow$ $S^{1}$ a 1-cocycle and suppose that the pair $\left(f_{\beta}, \beta\right)$ satisfies equation (4). The $f_{\beta}$ is termed a relative eigenfunction for $(X, \mu, G)$ over $(Y, \nu, G)$ with relative eigenvalue $\beta$.

Recall from [15] that relative eigenfunctions are of special interest in the calculation of those unitaries in $F(X, G)$ which normalize $F(Y, G)$. Indeed it is an easy calculation that if $f_{\gamma}$ is a relative eigenfunction with relative eigenvalue $\gamma$, the $T_{f_{\gamma}}$ is a unitary in $F(X, G)$ satisfying

$$
T_{f_{\gamma}}^{*}(m) T_{f_{\gamma}}=A_{\gamma}(m) \quad \forall m \in F(X, G)
$$

where $A_{\gamma}$ is the *-automorphism of $F(X, G)$ corresponding to the onecocycle $\gamma(\varphi(x), G)$. But since $\gamma$ is "restricted" from a cocycle on $Y \times G$, we see that

$$
A_{\gamma}\left(\varphi^{*}(F(Y, G))\right)=\varphi^{*}(F(Y, G)) .
$$

Hence $T_{f_{\gamma}}$ and $T_{f_{\gamma}}^{*}$ normalize $\varphi^{*}(F(Y, G))$. The main theorem of this section states that modulo multiplication by a unitary in $F(Y, G)$, every unitary in $F(X, G)$ normalizing $F(Y, G)$ must be of the form $T_{f_{\gamma}}$ for some relative eigenfunction $f_{\gamma}$. (Here we have identified $F(Y, G)$ with $\varphi^{*}(F(Y, G))$.) This will imply

THEOREM 2.2. Let $(X, \mu, G)$ be a free ergodic $G$-space, and suppose that $(Y, \nu, G)$ is a free quotient action, where $\mu$ is relatively G-invariant over $\nu$. Then the unitaries in $F(X, G)$ which normalize $F(Y, G)$ generate the von Neumann algebra $F\left(Z_{e}, G\right)$, where $\left(Z_{e}, \tau_{e}, G\right)$ is the maximal quotient action of $(X, \mu, G)$ having relatively elementary spectrum over $(Y, \nu, G)$. 
Before proving the theorem we make several introductory remarks about fibred product $G$-spaces.

Let $X \times{ }_{Y} X=\left\{\left(x_{1}, x_{2}\right) \in X \times X \mid \varphi\left(x_{1}\right)=\varphi\left(x_{2}\right)\right\}$. Define a measure $\mu_{F}$ on $X \times{ }_{Y} X$ by

$$
\mu_{F}=\int_{Y} \mu_{y} \times \mu_{y} d \nu \quad\left(\text { where } \mu=\int_{Y} \mu_{y} d \nu\right) .
$$

For example, note that if $(X, \mu)$ is a product space $(Y \times D, \nu \times m)$, then $\left(X \times{ }_{Y} X, \mu_{F}\right)$ is just the product measure space $(Y \times D \times D, \nu \times m \times m)$. Define an action of $G$ on $\left(X \times_{Y} X, \mu_{F}\right)$ by setting $\left(x_{1}, x_{2}\right) g=\left(x_{1} g, x_{2} g\right)$. The system $\left(X \times_{Y} X, \mu_{F}, G\right)$ will be ergodic if and only if $(X, \mu, G)$ is relatively weak-mixing over $(Y, \nu, G)$, i.e. if and only if the maximal quotient action of $(X, \mu, G)$ with relatively discrete spectum over $(Y, \nu, G)$ is $(Y, \nu, G)$ itself.

Examine the following commutative diagram of $G$-spaces:

$$
\begin{aligned}
& \left(X \times_{Y} X, \mu_{F}, G\right) \\
& \varphi_{1} \swarrow \\
& (X, \mu, G) \quad \downarrow \varphi_{3} \quad(X, \mu, G) \\
& \varphi \searrow \quad \swarrow \varphi \\
& (Y, \nu, G)
\end{aligned}
$$

Here

$$
\begin{aligned}
& \varphi_{1}\left(x_{1}, x_{2}\right)=x_{1} \\
& \varphi_{2}\left(x_{1}, x_{2}\right)=x_{2} \\
& \varphi_{3}\left(x_{1}, x_{2}\right)=\varphi\left(x_{1}\right)=\varphi\left(x_{2}\right) .
\end{aligned}
$$

Since $\varphi_{i} i=1,2,3$, and $\varphi$ are surjective $G$-maps, and since $(Y, \nu, G)$ and $(X, \mu, G)$ are ergodic, by applying [15], Proposition 1.1 the commutative diagram of von Neumann algebra injections can be constructed:

$$
\begin{aligned}
& F\left(X \times{ }_{Y} X, G\right) \\
& \varphi_{1}^{*} \nearrow \quad \nwarrow \varphi_{2}^{*} \\
& F(X, G) \quad \uparrow \varphi_{3}^{*} \quad F(X, G) \\
& \varphi^{*} \nwarrow \quad \nearrow \varphi^{*} \\
& F(Y, G)
\end{aligned}
$$

By the diagram we see that $\varphi_{1}^{*} \varphi^{*}(m)=\varphi_{2}^{*} \varphi^{*}(m)=\varphi_{3}^{*}(m) \forall m \in F(Y, G)$. Therefore with no confusion $F(Y, G)$ can be identified with its image in $F\left(X \times{ }_{Y} X, G\right)$. Also

$$
\varphi_{1}^{*}(F(X, G)) \cap \varphi_{2}^{*}(F(X, G))=F(Y, G) .
$$


For if

$$
\sum_{g \in G} T_{f_{g}} U_{g} \in \varphi_{1}^{*}(F(X, G)) \cap \varphi_{2}^{*}(F(X, G)),
$$

where $f_{g} \in L^{\infty}\left(X \times{ }_{Y} X\right)$, then each $f_{g}$ is constant along the fibers of both $\varphi_{1}$ and $\varphi_{2}$. But this implies that each function $f_{g}$ is constant along the fibers of $\varphi_{3}$, hence $\sum_{g \in G} T_{f_{g}} U_{g} \in \varphi_{3}^{*}(F(Y, G))=F(Y, G)$. We are now prepared for the proof of the main theorem.

Proof of Theorem 2.2. Suppose $U$ is a unitary element in $F(X, G)$ such that $U F(Y, G) U^{*}=F(Y, G)$. We examine the element $\varphi_{1}^{*}(U) \varphi_{2}^{*}\left(U^{*}\right)=$ $K \in F\left(X \times{ }_{Y} X, G\right)$. We claim that $K$ commutes with everything in $F(Y, G)$. Indeed for $m \in F(Y, G)$,

$$
\begin{aligned}
K \varphi_{3}^{*}(m) K^{*} & =\varphi_{1}^{*}(U) \varphi_{2}^{*}\left(U^{*}\right) \varphi_{2}^{*}\left(\varphi^{*}(m)\right) \varphi_{2}^{*}(U) \varphi_{1}^{*}\left(U^{*}\right) \\
& =\varphi_{1}^{*}(U) \varphi_{2}^{*}\left(U^{*} \varphi^{*}(m) U\right) \varphi_{1}^{*}\left(U^{*}\right) \\
& =\varphi_{1}^{*}(U) \varphi_{1}^{*}\left(\varphi^{*}\left(\varphi^{*-1}\left(U^{*} \varphi^{*}(m) U\right)\right)\right) \varphi_{1}^{*}\left(U^{*}\right) \\
& =\varphi_{1}^{*}\left(U \varphi^{*}\left(\varphi^{*-1}\left(U^{*} \varphi^{*}(m) U\right)\right) U^{*}\right) \\
& =\varphi_{1}^{*}\left(U U^{*} \varphi^{*}(m) U U^{*}\right)=\varphi_{1}^{*}\left(\varphi^{*}(m)\right)=\varphi_{3}^{*}(m)
\end{aligned}
$$

Therefore $K \varphi_{3}^{*}(m)=\varphi_{3}^{*}(m) K \forall m \in F(Y, G)$. In particular $K$ commutes with everything in $R(Y, G)$. Since $(Y, \nu, G)$ is a free action and $\mu$ is relatively $G$-invariant over $\nu$, by Lemma $1.8 K \in R\left(X \times{ }_{Y} X, G\right)$. Since $K$ is unitary, we can write

$$
K=T_{\gamma}, \quad \text { where } \gamma \in L^{\infty}\left(X \times{ }_{Y} X\right),|\gamma|=1 .
$$

We now give $U$ its unique representation $U=\sum_{g \in G} T_{f_{g}} U_{g}$, with $f_{g} \in$ $L^{\infty}(X) \forall g \in G$. Then

$$
\varphi_{1}^{*}(U)=\sum_{g \in G} T_{f_{g, 1}} U_{g} \text { and } \varphi_{2}^{*}(U)=\sum_{g \in G} T_{f_{g, 2}} U_{g}
$$

with

$$
\begin{array}{ll}
f_{g, 1}\left(x_{1}, x_{2}\right)=f_{g}\left(x_{1}\right), & g \in G \\
f_{g, 2}\left(x_{1}, x_{2}\right)=f_{g}\left(x_{2}\right), & g \in G .
\end{array}
$$
implies

Since $\varphi_{1}^{*}(U)=K \varphi_{2}^{*}(U)$, we obtain $\Sigma_{g \in G} T_{f_{g, 1}} U_{g}=T_{\gamma} \sum_{g \in G} T_{f_{g, 2}} U_{g}$ which

$$
f_{g, 1}=\gamma f_{g, 2} \quad \mu_{F} \text { a.e., } \forall g \in G \text {. }
$$


Therefore

$$
T_{\left|f_{g, 1}\right|}=T_{|\gamma|\left|f_{g, 2}\right|}=T_{\left|f_{g, 2}\right|} \text { since }|\gamma|=1 .
$$

Recalling that $\varphi_{1}^{*}(F(X, G)) \cap \varphi_{2}^{*}(F(X, g))=F(Y, G)$, we obtain $\left|f_{g, 2}\right|=$ $\left|f_{g, 2}\right| \in \varphi^{*}\left(L^{\infty}(Y)\right)$ so that $\left|f_{g}(x)\right|$ is constant along the fibers of $\varphi$, $\forall g \in G$.

Setting $Z_{g}=\left\{x \in X \mid f_{g}(x)=0\right\}$, it is clear that $Z_{g}$ is a lift of a measurable set in $(Y, \nu)$. Hence $A_{g}=X-Z_{g}$ is also a lift of a measurable set in $Y$.

Now $\forall k, g \in G$, define

$$
R_{k, g}(x)= \begin{cases}\left|f_{g}(x)\right| \frac{f_{k}(x)}{f_{g}(x)} & x \in A_{g}, \\ 0 & x \in Z_{g} .\end{cases}
$$

Clearly $R_{k, g}(x) \in L^{\infty}(X)$. We claim $R_{k, g}$ is constant along the fibers of $\varphi$.

We have already seen that $\left|f_{g}\right|$ is constant along the fibers of $\varphi$, and for $\left(x_{1}, x_{2}\right) \in X \times \times_{Y} X \cap A_{g} x A_{g}$,

$$
\frac{f_{k}\left(x_{1}\right)}{f_{g}\left(x_{1}\right)}=\frac{\gamma\left(x_{1}, x_{2}\right) f_{k}\left(x_{2}\right)}{\gamma\left(x_{1}, x_{2}\right) f_{g}\left(x_{2}\right)}=\frac{f_{k}\left(x_{2}\right)}{f_{g}\left(x_{2}\right)} \text {. }
$$

Equation (11) comes from dividing through equation (9) with $g=k$ through by equation (9) on the support of $f_{g}$.

Therefore $\forall(k, g) \in G \times G$,

$$
R_{k, g}(x)=r_{k, g}(\varphi(x)) \quad \text { where } r_{k, g} \in L^{\infty}(Y) .
$$

We now claim that we can write

$$
U=T_{\tau(x)} \sum_{g \in G} T_{m_{g} \circ \varphi} U_{g}
$$

where $m_{g} \in L^{\infty}(Y)$ and $\tau \in L^{\infty}(X),|\tau| \equiv 1$.

First, note that $\mu\left(X-\cup_{g \in G} A_{g}\right)=0$, as the identity $U U^{*}=0 \Rightarrow$ $\sum_{g \in G} f_{g}(x) \overline{f_{g}(x)}=1 \mu$ a.e. Using the fact that $G$ is countable, we enumerate it in some fashion: $G=\left\{g_{i} \mid i=1,2, \ldots\right\}$. Set $B_{1}=A_{g_{1}}$, and for $i>1$, set

$$
B_{i}=A_{g_{t}} \cap Z_{g_{t-1}} \cap \cdots \cap Z_{g_{1}} .
$$

Then the collection $\left\{B_{l} \mid i \in \mathbf{N}\right\}$ are mutually disjoint sets whose union is equal to $\bigcup_{g \in G} A_{g}=X \mu$ a.e. Furthermore each $B_{i}$ is a lift of a measurable subset of $Y$. For each $k \in G$, examine

$$
M_{k}(x)=\sum_{i=i} R_{k g_{i}}(x) \chi_{B_{i}}(x) .
$$


Clearly $M_{k}(x)$ is constant along the fibers of $\varphi$, hence $M_{k}(x)=m_{k}(\varphi(x))$ $\mu$ a.e. for some $m_{k} \in L^{\infty}(Y)$.

Now define

$$
\tau(x)=\sum_{i=1}^{\infty} \frac{f_{g_{i}}(x)}{\left|f_{g_{i}}(x)\right|+\chi_{z_{g_{i}}}(x)} \chi_{B_{i}}(x) .
$$

That $|\tau(x)|=1 \mu$ a.e. follows from the fact that the $B_{i}$ are mutually disjoint with $\mu\left(X-\cup_{i=1}^{\infty} B_{i}\right)=0$.

We claim that

$$
\tau(x) M_{k}(x)=f_{k}(x) \quad \mu \text { a.e., } \quad \forall k \in G .
$$

Indeed

$$
\begin{aligned}
\tau(x) M_{k}(x) & =\left(\sum_{i=1}^{\infty} \frac{f_{g_{i}}(x)}{\left|f_{g_{i}}(x)\right|+\chi_{Z_{g_{i}}}(x)} \cdot \chi_{B_{i}}(x)\right)\left(\sum_{i=1}^{\infty} R_{k, g_{i}}(x) \chi_{B_{i}}(x)\right) \\
& =\sum_{i=1}^{\infty}\left(\frac{f_{g_{i}}}{\left|f_{g_{i}}(x)\right|+\chi_{Z_{g_{i}}}(x)} R_{k, g_{i}}(x)\right) \chi_{B_{i}} \\
& =\sum_{i=1}^{\infty}\left(\frac{f_{g_{i}}(x)}{\left|f_{g_{i}}(x)\right|} \cdot\left|f_{g_{i}}(x)\right| \frac{f_{k}(x)}{f_{g_{i}}(x)} \chi_{A_{g_{i}}}\right) \chi_{B_{i}} \\
& =\sum_{i=1}^{\infty} f_{k}(x) \chi_{B_{i}}=f_{k}(x) \sum_{i=1}^{\infty} \chi_{B_{i}}=f_{k}(x) \quad \mu \text { a.e. }
\end{aligned}
$$

Therefore

$$
\begin{aligned}
U & =\sum_{g \in G} T_{\tau(x) M_{g}(x)} U_{g}=T_{\tau(x)} \sum_{g \in G} T_{M_{g}(x)} U_{g} \\
& =T_{\tau} \sum_{g \in G} T_{m_{g} \circ \varphi} U_{g}=T_{\tau} \varphi^{*}\left(\sum_{g \in G} T_{m_{g}} U_{g}\right) .
\end{aligned}
$$

Since $T_{\tau}$ is unitary in $F(X, G), \sum_{g \in G} T_{m_{g}} U_{g}$ is unitary in $F(Y, G)$. Furthermore, since $U$ normalizes $\varphi^{*}(F(Y, G))$ by hypothesis and $\varphi^{*}\left(\sum_{g \in G} T_{m_{g}} U_{g}\right)$ obviously normalizes $\varphi^{*}(F(Y, G))$, it is clear that $T_{\tau}$ must normalize $\varphi^{*}(F(Y, G))$. In particular $T_{\tau}^{*} U_{g} T_{\tau} \in \varphi^{*}(F(Y, G)), \forall g \in G$. This implies that $\tau(x g) \overline{\tau(x)}$ is constant along the fibers of $\varphi$, so that $\tau(x g) \overline{\tau(x)}=$ $\rho(\varphi(x), g)$ for some $\rho: Y \times G \rightarrow S^{1}$. Clearly $\rho$ satisfies the cocycle identity. Therefore $\tau$ is a relative eigenfunction for $(X, \mu, G)$ over $(Y, \nu, G)$ with relative eigenvalue $\rho$. Therefore $U \in F\left(Z_{e}, G\right)$, and the remarks about relative eigenfunctions made preceding the theorem show that 
$F\left(Z_{e}, G\right)$ is contained in the subalgebra of $F(X, G)$ generated by those unitaries which normalize $F(Y, G)$. This completes the proof.

We state several corollaries of Theorem 2.2; the first provides the promised generalization of the remarks made following Theorem 3.3 of [15]:

COROllary 2.3. Let $(X, \mu, G)$ and $(Y, \nu, G)$ be as in the statement of Theorem 2.5. Then there exists a compact second countable abelian group $A$, and a cocycle $\beta: Y \times G \rightarrow A$, such that $\left(Y \times{ }_{\beta} A, \nu \times \nu_{A}, G\right)$ is the maximal quotient action of $(X, \mu, G)$ having relatively elementary spectrum over $(Y, \nu, G)$, and such that every unitary operator in $F(X, G)$ which normalizes $F(Y, G)$ is of the form $T_{f_{\chi}} W$, for some $\chi \in \hat{A}$ and $W \in \mathscr{U}(F(Y, G))$. (Here $f_{\chi}(y, a)=\chi(a)$.)

Proof. In the course of proving Theorem 2.2 it was shown that any unitary normalizing $F(Y, G)$ must be of the form $T_{\tau} W, W \in U(F(Y, G))$, $\tau$ a relative eigenfunction for $(X, \mu, G)$ over $(Y, \nu, G)$. Zimmer's results on relatively elementary spectrum in [27], [4] show that $\left(Z_{e}, G\right)=\left(Y \times{ }_{\beta} A\right.$, $G)$ for some compact second countable abelian group $A$ and cocycle $\beta$ : $Y \times G \rightarrow A$. Also by results of [27] the decomposition of the natural cocycle bundle representation on $Y \times G$ induced from the identity on $Y \times{ }_{\beta} A \times G$ into one-dimensional subbundle representations is unique up to equivalence. Thus for any relative eigenfunction $\tau$ there exists $\chi \in \hat{A}$ and Borel $b: Y \rightarrow S^{1}$ with $\tau=f_{\chi} b$. But then $T_{\tau} W=T_{f_{x} b} W=T_{f_{x}} T_{b} W$, which is of the desired form.

Corollary 2.4. Let $(X, \mu, G)$ and $(Y, \nu, G)$ be as in Theorem 2.2. The unitaries in $F(X, G)$ normalizing $F(Y, G)$ will generate $F(X, G)$ if and only if $(X, \mu, G)$ has relatively elementary spectrum over $(Y, \nu, G)$.

With the theorem in hand we are now able to calculate the "length" of $F(Y, G)$ in $F(X, G)$. We briefly review the relevant definitions, the first of which was introduced in [23].

Definition 2.5. Let $\mathscr{A}$ be a von Neumann subalgebra of the von Neumann algebra $\mathscr{M}$. Set $\mathscr{A}=N_{0}(\mathscr{A})$ and proceed inductively, setting $N_{i+1}(\mathscr{A})=$ the von Neumann algebra generated by $\{U$ unitary in $\left.\mathscr{M} \mid U^{*} N_{i}(\mathscr{A}) U=N_{i}(\mathscr{A})\right\}$. Call $N_{1}(\mathscr{A})$ the normalizer of $\mathscr{A}$ in $\mathscr{M}$. Then

$$
N_{0}(\mathscr{A})=\mathscr{A} \subseteq N_{1}(\mathscr{A}) \subseteq \cdots \subseteq N_{i}(\mathscr{A}) \text {. }
$$


Define the finite length of $\mathscr{A}$ in $\mathscr{M}$ to be the least positive integer $n$ such that $N_{n}(\mathscr{A})=N_{n+1}(\mathscr{A})$, if such an $n$ exists. Call the length of $\mathscr{A}$ infinite if no such $n$ exists.

When the length of $\mathscr{A}$ in $\mathscr{M}$ is infinite, the notion of transfinite length must be introduced:

Definition 2.6. Let $\mathscr{A}$ and $\mathscr{M}$ be as in 2.5, and suppose that the length of $\mathscr{A}$ in $\mathscr{M}$ is infinite. Let $N_{\omega_{0}}(\mathscr{A})$ be the von Neumann subalgebra of $\mathscr{M}$ generated by $\bigcup_{i \in N^{+}} N_{i}(\mathscr{A})$. Proceed on in this way, defining for any non-limit countable ordinal $\eta N_{\eta}(\mathscr{A})=N_{1}\left(N_{\eta-1}(\mathscr{A})\right.$ ), and for $\eta$ a limit ordinal, define $N_{\eta}(\mathscr{A})$ to be the von Neumann algebra generated by $\bigcup_{\xi<\eta} N_{\xi}(\mathscr{A})$. Define the transfinite length of $\mathscr{A}$ in $\mathscr{M}$ to the least countable ordinal $\eta$ such that $N_{\eta}(\mathscr{A})=N_{\eta+1}(\mathscr{A})$ if such a countable ordinal exists.

Note that if $\mathscr{A}$ has finite length in $\mathscr{M}$, the transfinite length of $\mathscr{A}$ in $\mathscr{M}$ will coincide with the finite length of $\mathscr{A}$ in $\mathscr{M}$, therefore without loss of generality we refer to the transfinite length of $\mathscr{A}$ in $\mathscr{M}$ as the length of $\mathscr{A}$ in $\mathscr{M}$.

We now wish to calculate the length of $F(Y, G)$ in $F(X, G)$ under the conditions of Theorem 2.2, and furthermore to relate the calculation to Zimmer's notion of generalized elementary spectrum [28]. Recall that the ergodic $G$-space $(X, \mu, G)$ is said to have generalized elementary spectrumover $(Y, \nu, G)$ if it can be built up from $(Y, \nu, G)$ by taking extensions with relatively elementary spectrum and inverse limits. To each such extension $(X, \mu, G)$ of $(Y, \nu, G)$ is associated a countable ordinal $\alpha$, which, roughly speaking, represents the minimum number of steps needed to construct $(X, \mu, G)$. The following theorem relates generalized elementary spectrum to the length of $F(Y, G)$ in $F(X, G)$ :

TheOREM 2.7. Let $(X, \mu, G)$ be a free ergodic action of the countable group $G$ on the compact Lebesgue space $(X, \mu)$, and let $(Y, \nu, G)$ be a free quotient action, with $\mu$ relatively $G$-invariant over $\nu=\varphi_{*} \mu$, where $\varphi: X \rightarrow Y$ is the quotient $G$-map. Then the length of $F(Y, G)$ in $F(X, G)$ is equal to $\alpha$, and $N_{\alpha}(F(Y, G))=F\left(Z_{g e}, G\right)$, where $\left(Z_{g e}, \tau_{g e}, G\right)$ is the maximal quotient action of $(X, \mu, G)$ having generalized elementary spectrum over $(Y, \nu, G)$, and $\alpha$ is the ordinal of this spectrum.

Proof. By Zimmer's theory, there exists a countable ordinal $\alpha$, a chain of extensions $\left\{\left(X_{\gamma}, \mu_{\gamma}, G\right) \mid \gamma(\alpha)\right\}$ with $\left(X_{0}, \mu_{0}, G\right)=(Y, \nu, G)$ and 
$\left(X_{\alpha}, \mu_{\alpha}, G\right)=\left(Z_{g e}, \tau_{g e}, G\right)=\left(X_{\alpha}, \mu_{\alpha}, G\right)$. These extensions are chosen in such a fashion that if $\gamma$ is not a limit ordinal, then $\left(X_{\gamma+1}, \mu_{\gamma+1}, G\right)$ is the maximal extension of $\left(X_{\gamma}, \mu_{\gamma}, G\right)$ which is also a quotient action of $(X, \mu, G)$ having relatively elementary spectrum over $\left(X_{\gamma}, \mu_{\gamma}, G\right)$, and if $\gamma$ is a limit ordinal, then $\left(X_{\gamma}, \mu_{\gamma}, G\right)=\operatorname{inj} \lim _{\eta<\gamma}\left(X_{\eta}, \mu_{\eta}, G\right)$. We prove the theorem by induction, showing that

$$
N_{\xi}(F(Y, G))=F\left(X_{\xi}, G\right) \quad \forall \xi \leq \alpha .
$$

The case $\xi=0$ is obvious from the definition. Assume that $(*)$ is true for every ordinal less than some fixed $\xi<\alpha$. If $\xi$ is not a limit ordinal, then

$$
N_{\xi}(F(Y, G))=N_{1}\left(N_{\xi-1}(F(Y, G))\right)=N_{1}\left(F\left(X_{\xi-1}, G\right)\right),
$$

and by Theorem $2.2, N_{1}\left(F\left(X_{\xi-1}, G\right)\right)=F\left(X_{\xi}, G\right)$. If $\xi$ is a limit ordinal, the $N_{\xi}(F(Y, G))$ is equal to the von Neumann algebra generated by $\bigcup_{\gamma<\xi} F\left(X_{\gamma}, G\right)$. Therefore our aim is to show that the von Neumann subalgebra of $F(X, G)$ generated by $\bigcup_{\gamma<\xi} F\left(X_{\gamma}, G\right)$ is equal to $F\left(\operatorname{inj} \lim _{\gamma<\xi}\left(X_{\gamma}, G\right)\right)=F\left(X_{\xi}, G\right)$. But each $F\left(X_{\gamma}, G\right)$ is generated by $\left\{U_{g} \mid g \in G\right\}$ and $R\left(X_{\gamma}, G\right)$. Therefore $N_{\xi}(F(Y, G))$ is generated by $\left\{U_{g} \mid g\right.$ $\in G\}$ and $\bigcup_{\gamma<\xi} R\left(X_{\gamma}, G\right)$. Each $R\left(X_{\gamma}, G\right)$ is *-isomorphic to $L^{\infty}\left(X_{\gamma}, \mu_{\gamma}\right)$, and the subalgebra generated by $\bigcup_{\gamma<\xi} L^{\infty}\left(X_{\gamma}, \mu_{\gamma}\right)$ is precisely $L^{\infty}\left(X_{\xi}, \mu_{\xi}\right)$ by the properties of inverse limits of $G$-spaces (see [28], Section 8). Hence the subalgebra generated by $\bigcup_{\gamma<\xi} R\left(X_{\gamma}, G\right)$ is $R\left(X_{\xi}, G\right)$. Hence $N_{\xi}(F(Y, G))$ is equal to the von Neumann algebra generated by $\left\{U_{g} \mid g \in\right.$ $G\}$ and $R\left(X_{\xi}, G\right)$, and thus we obtain, for every limit ordinal $\xi \leq \alpha$, $N_{\xi}(F(Y, G))=F\left(X_{\xi}, G\right)$. By induction, this completes the proof.

Assume now that $G$ is abelian and preserves the finite normalized measure $\mu$. In [15], we proved that $N_{1}(S(X, G))=F\left(Y_{d}, G\right)$, where $\left(Y_{d}, \tau, G\right)$ is the maximal quotient action of $(X, \mu, G)$ having pure point spectrum. If $\left(Y_{d}, \tau, G\right)$ is a free action, we shall be able to use Theorem 2.2 to find $N_{1}\left(F\left(Y_{d}, G\right)\right)=N_{2}(S(X, G)), N_{2}\left(F\left(Y_{d}, G\right)\right)$, and so forth. The subalgebra obtained by a transfinite continuation of this procedure corresponds to the maximal quotient action of $(X, \mu, G)$ having what is called generalized elementary spectrum:

Definition 2.8. Let $(X, \mu, G)$ be an ergodic $G$-space. Then $(X, \mu, G)$ is said to have generalized elementary spectrum of ordinal $\alpha$ if $(X, \mu, G)$ has generalized elementary spectrum over $\left(Y_{0}, \nu_{0}, G\right)$ of ordinal $\alpha$, where $\left(Y_{0}, \nu_{0}, G\right)$ represents the trivial ergodic action of $G$ on a point. 
The following corollary follows immediately from Theorem 2.3 of [15] and Theorem 2.7.

CoROllary 2.9. Let $(X, \mu, G)$ be a free ergodic action of the countable abelian group $G$ on the compact Lebesgue space $(X, \mu)$ which preserves the finite measure $\mu$, such that the maximal quotient action of $(X, \mu, G)$ with pure point spectrum is a free action. Then the length of $S(X, G)$ in $F(X, G)$ is equal to $\eta$, and $N_{\eta}(S(X, G))=F\left(Z_{g e}, \tau_{g e}, G\right)$ where $\left(Z_{g e}, \tau, G\right)$ is the maximal quotient action of $(X, \mu, G)$ having generalized elementary spectrum, and $\eta$ is equal to the ordinal of this spectrum.

EXAMPLES. Let $X_{n}=T^{n}$, the $n$-torus, with Haar measure $\mu_{n}$, and let an action of $\mathbf{Z}$ on $\left(X_{n}, \mu_{n}\right)$ be generated by the transformations $\left(z_{1}, \ldots, z_{n}\right)$ $\mapsto\left(\lambda z_{1}, z_{1} z_{2}, z_{n-1} z_{n}\right)$, where $\lambda=e^{2 \pi i \alpha}, \alpha$ irrational. Then $\left(X_{n}, \mu_{n}, \mathbf{Z}\right)$ has generalized elementary spectrum of ordinal $n$, where the chain of quotient actions is given by $\left\{\left(X_{i}, \mu_{i}, \mathbf{Z}\right) \mid i \leq n\right\}$. Hence the length of $S\left(X_{n}, \mathbf{Z}\right)$ in $F\left(X_{n}, \mathbf{Z}\right)$ is equal to $n$, and $N_{n}\left(S\left(X_{n}, \mathbf{Z}\right)\right)=F\left(X_{n}, \mathbf{Z}\right)$.

If $(Y, \nu, Z)$ is an arbitrary weak-mixing $\mathbf{Z}$-space (such as a Bernoulli shift), then form the product action $\left(X_{n} \times Y, \mu_{n} \times \nu, Z\right)$ given by $(x, y) n$ $=(x \cdot n, y \cdot n)$. Call this action $\left(Z_{n}, \tau_{n}, \mathbf{Z}\right)$. Then $\left(Z_{n}, \tau_{n}, \mathbf{Z}\right)$ has as its maximal quotient action with generalized elementary spectrum $\left(X_{n}, \mu_{n}, \mathbf{Z}\right)$. Thus the length of $S\left(Z_{n}, \mathbf{Z}\right)$ in $F\left(Z_{n}, \mathbf{Z}\right)$ is still $n$, but $N_{n}\left(S\left(Z_{n}, \mathbf{Z}\right)\right) \cong$ $F\left(X_{n}, \mathbf{Z}\right)$. (Note that $\left(Z_{n}, \tau, \mathbf{Z}\right)$ is relatively weak mixing over $F\left(X_{n}, \mathbf{Z}\right)$.)

Let $X_{\infty}=\prod_{i=1}^{\infty}\left(S^{1}\right)_{i}$, with Haar measure $\mu_{\infty}$. Then an ergodic action of $\mathbf{Z}$ on $X_{\infty}$ is generated by the transformation $\left(z_{1}, z_{2}, \ldots, z_{n}, \ldots\right) \mapsto$ $\left(\lambda z_{1}, z_{1} z_{2}, \ldots, z_{n-1} z_{n}, \ldots\right)$ where $\lambda$ is as above. Then

$$
\left(X_{\infty}, \mu_{\infty}, \mathbf{Z}\right)=\underset{\text { inj }}{ } \lim \left(X_{n}, \nu_{n}, \mathbf{Z}\right)
$$

and $\left(X_{\infty}, \mu_{\infty}, \mathbf{Z}\right)$ has generalized elementary spectrum of ordinal $\chi_{0}$, the first infinite ordinal. Hence $S\left(X_{\infty}, \mathbf{Z}\right)$ has length $\chi_{0}$ in $F\left(X_{0}, \mathbf{Z}\right)$ and $N_{\chi_{0}}\left(S\left(X_{\infty}, \mathbf{Z}\right)\right)=F\left(X_{\infty}, \mathbf{Z}\right)$. Forming $\left(Z_{\infty}, \tau_{\infty}, \mathbf{Z}\right)=\left(X_{\infty} \times Y, \mu_{\infty} \times \nu, \mathbf{Z}\right)$ where $(Y, \nu, Z)$ is as in the previous paragraph, then the length of $S\left(Z_{\infty}, \mathbf{Z}\right)$ in $F\left(Z_{\infty}, \mathbf{Z}\right)$ is still $\chi_{0}$, but $N_{\chi_{0}}\left(S\left(Z_{\infty}, \mathbf{Z}\right)\right) \cong F\left(X_{\infty}, \mathbf{Z}\right) \subsetneq$ $F\left(Z_{\infty}, \mathbf{Z}\right)$.

REMARK 2.10. We conjecture that when $G$ is abelian and preserves the finite measure $\mu$, Theorem 2.2 can be extended to the case where $(Y, \nu, G)$ is not a free action. 
3. The normalizer of $S(X, G)$ in $F(X, G)$ when $(X, \mu, G)$ is weak-mixing. In [13], Nielsen proved that if $(X, \mu, G)$ is a free ergodic $G$-space, where $G$ is countable and abelian, and if the $L^{\infty}$-spectrum of the $G$-action is trivial, then $S(X, G)$ is singular in $F(X, G)$ so that $N_{1}(S(X, G))=S(X, G)$. His proof used direct integral decomposition techniques and Takesaki's equivalence relation. In this section we provide an alternate proof of this fact for the case where $\mu$ is $G$-invariant, which is valid for $G$ non-abelian as well. The proof uses the embedding idea which was exploited in the previous section.

We recall some terminology and theory. Let $(X, \mu, G)$ be a free ergodic $G$-space where $\mu$ is finite and $G$-invariant. Then $(X, \mu, G)$ is called weak-mixing if the unitary representation of $G$ on $L^{2}(X) \ominus \mathbf{C}$ has no finite dimensional subrepresentations. A result of Moore [10, Proposition 1], which extends classical results for $G=\mathbf{R}$ or $\mathbf{Z}$, shows that $(X, \mu, G)$ is weak mixing if and only if the action of $G$ on $(X \times X, \mu \times \mu)$ given by $\left(x_{1}, x_{2}\right) g=\left(x_{1} g, x_{2} g\right)$ is ergodic.

Recall that when $G$ is abelian and $(X, \mu, G)$ is as above $S(X, G)$ is a maximal abelian subalgebra of $F(X, G)$. Clearly if $G$ is not abelian this will no longer be true. However, when $(X, \mu, G)$ is weak-mixing, one can show that any element in $F(X, G)$ which commutes with everything in $S(X, G)$ must lie in $S(X, G)$ itself:

Lemma 3.1. Let $(X, \mu, G)$ be a free ergodic $G$-space, where $G$ preserves the finite measure $\mu$, and suppose that $(X, \mu, G)$ is weak-mixing. Then $S(X, G)^{\prime} \cap F(X, G) \subset S(X, G)$.

Proof. Let $g \in G$. Set $H_{g}=\left\{h \in G \mid h g h^{-1}=G\right\}$. We claim that if $g$ has finite conjugacy class in $G$, then $H_{g}$ must act ergodically on $(X, \mu, G)$. For if $H_{g}$ does not act ergodically on $(X, \mu)$ there exists a non constant function $f \in L^{\infty}(X) \subset L^{2}(X)$ with $f$ invariant under the action of $H_{g}$. If $g_{1}, g_{2}$ are in the same right $H_{g}$ coset, the $f\left(x g_{1}\right)=f\left(x g_{2}\right)$ since $g_{1}=g_{2} h$ for some $h \in H_{g}$. Since $\left[G: H_{g}\right]=\operatorname{card}\left\{h g h^{-1} \mid h \in G\right\}$ which is finite, the subspace of $L^{2}(X)$ spanned by $\{f(x g) \mid g \in G\}$ is finite dimensional, and the representation of $G$ on $L^{2}(X) \ominus C$ has a finite-dimensional subrepresentation, which is a contradiction of our original assumption.

Now suppose that $Q \in F(X, G)$ commutes with everything in $S(X, G)$. Then write

$$
Q=\sum_{g \in G} T_{f_{g}} U_{g}, \quad \text { with } f_{g} \in L^{\infty}(X) \forall g \in G
$$


For every $h \in G$,

$$
\begin{aligned}
U_{h} Q & =Q U_{h} \Rightarrow U_{h} \sum_{g \in G} T_{f_{g}} U_{g}=\sum_{g \in G} T_{f_{g}} U_{g} U_{h} \\
& \Rightarrow \sum_{g \in G} T_{f_{g}(x h)} U_{h g}=\sum_{g \in G} T_{f_{g}} U_{g h} \\
& \Rightarrow \sum T_{f_{g}(x h)} U_{h g}=\sum_{g \in G} T_{f_{h g h}-1} U_{h g} \\
& \Rightarrow f_{g}(x h)=f_{h g h^{-1}}(x) \quad \mu \text { a.e., } \quad \forall g \in G, \forall h \in G .
\end{aligned}
$$

We now claim that if $g$ has an infinite number of conjugates in $G$, we must have $f_{g}(x) \equiv 0$ in $L^{\infty}(X)$. For if this were not the case there would be an infinite number of functions

$$
\left\{f_{h^{-1} g h}(x) \mid h \in G\right\}
$$

with

$$
\int_{X}\left|f_{h^{-1} g h}(x)\right|^{2} d \mu=\int\left|f_{g}(x h)\right|^{2} d \mu=\int\left|f_{g}(x)\right|^{2} d \mu=K>0
$$

since $G$ preserves $\mu$, but then $\sum_{g \in G}\left\|f_{g}(x)\right\|_{L^{2}}=\infty$ which is impossible. Therefore if $Q \in(S(X, G))^{\prime} \cap F(X, G)$ we must have $Q=\sum_{g \in G_{0}} T_{f_{g}} U_{g}$, where $G_{0}$ is the subset of $G$ consisting of those $g$ lying in finite conjugacy classes. Furthermore

$$
f_{g}(x h)=f_{h^{-1} g h}(x) \quad \mu \text { a.e., } \quad \forall h \in G .
$$

But if $h \in H_{g}$, we obtain $f_{g}$ is $H_{g}$ invariant, $\forall g \in G_{0}$. This implies that $f_{g}$ is a constant function since $H_{g}$ acts ergodically on $(X, \mu, G)$. Therefore $Q \in S(X, G)$, and this completes the proof.

REMARK 3.2. If $(X, \mu, G)$ is a free ergodic $G$-space, and if there exists $g \in G_{0}$ such that $H_{g}$ does not act ergodically on $(X, \mu)$, it is easy to construct an element in $S(X, G)^{\prime} \cap F(X, G)$ which is not in $S(X, G)$.

We now proceed to the proof of the main theorem of this section.

THeOREM 3.3. Let $(X, \mu, G)$ be a free ergodic action of the countable group $G$ on a compact Lebesgue space which preserves the finite measure $\mu$ and which is weak-mixing. Then any unitary in $F(X, G)$ which normalizes $S(X, G)$ lies in $S(X, G)$ so that $N_{1}(S(X, G))=S(X, G)$. 
Proof. Form the product $G$-space $(X \times X, \mu \times \mu, G)$. As in the proof of Theorem 2.2 we have quotient $G$ maps

$$
\begin{aligned}
& (X \times X, \mu \times \mu, G)
\end{aligned}
$$

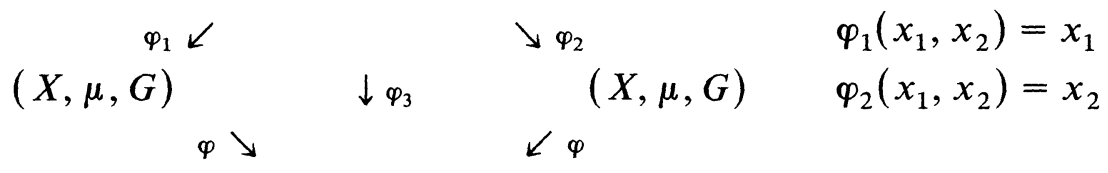

$$
\begin{aligned}
& (Y, \nu, G) \text { where } Y=\text { pt. }
\end{aligned}
$$

Then as in the proof of Theorem 2.2, there are associated injections of von Neumann algebras:

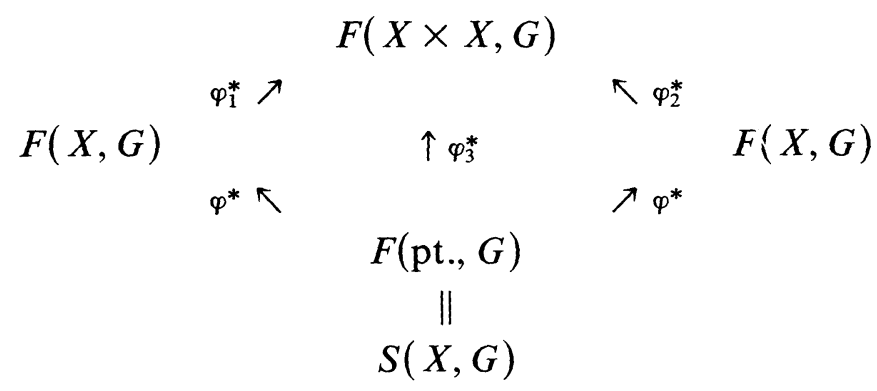

with $\varphi_{2}^{*}(F(X, G)) \cap \varphi_{1}^{*}(F(X, G))=\varphi^{*}(S(X, G))=S(X \times X, G)$.

Suppose that $U$ unitary in $F(X, G)$ normalizes $S(X, G)$. Then $\varphi_{1}^{*}(U) \varphi_{2}^{*}\left(U^{*}\right)$ commutes with everything in $\varphi^{*}(S(X, G))=S(X \times X, G)$. Since $(X, \mu, G)$ is weak mixing, it is not hard to see that $(X \times X, \mu \times \mu$, $G)$ is weak-mixing. (See [28], Cor. 7.11). Hence $\varphi_{1}^{*}(U) \varphi_{2}^{*}\left(U^{*}\right) \in$ $S(X \times X, G)$ by Lemma 3.1. This implies that $\varphi_{1}^{*}(U)$ and $\varphi_{2}^{*}(U)$ are both elements of $\varphi_{1}^{*}(F(X, G)) \cap \varphi_{2}^{*}(F(X, G))=\varphi_{3}^{*}(S(X, G))$. Hence $U \in$ $S(X, G)$, as desired.

The same methods can be applied to prove the following partial generalization of Theorem 2.2:

THEOREM 3.4. Let $(X, \mu, G)$ be a free ergodic $G$-space and suppose that $(Y, \nu, G)$ is a quotient $G$-space (but not necessarily free) where $\mu$ is finite relatively $G$-invariant over $\nu,(X, \mu, G)$ is relatively weak-mixing over $(Y, \nu, G)$, and $G$ is abelian. Then $N_{1}(F(Y, G))=F(Y, G)$.

Proof. As in the proof of Theorem 2.2, form the diagram given in (7) of §2. Again $\varphi_{1}^{*}(F(X, G)) \cap \varphi_{2}^{*}(F(X, G))=\varphi^{*}(F(Y, G))$. If $U$ normalizes $F(Y, G)$, then $\varphi_{1}^{*}(U) \varphi_{2}^{*}\left(U^{*}\right)$ commutes with everything in $\varphi^{*}(S(Y, G))=$ $S\left(X \times_{Y} X, G\right)$. Since $\left(X \times_{Y} X, \mu_{F}, G\right)$ is ergodic $S\left(X \times_{Y} X, G\right)$ is 
maximal abelian. Therefore

$$
\varphi_{1}^{*}(U) \varphi_{2}^{*}\left(U^{*}\right) \in S\left(X \times_{Y} X, G\right) \subset \varphi^{*}(F(Y, G)),
$$

which implies that $\varphi_{1}^{*}(U)$ and $\varphi_{2}^{*}(U)$ are both in $\varphi_{1}^{*}(F(X, G)) \cap$ $\varphi_{2}^{*}(F(X, G))$. Hence $U \in F(Y, G)$, as desired.

The following is an application of Theorem 3.4.

COROllary 3.5. Let $(X, \mu, G)$ be a free ergodic action where the abelian group $G$ preserves $\mu$, and suppose that the maximal quotient action of $(X, \mu, G)$ with pure point spectrum, say $\left(Y_{d}, \nu, G\right)$, is a transitive action. Then the length of $S(X, G)$ in $F(X, G)$ is one, with $N_{1}(S(X, G))=$ $F\left(Y_{d}, G\right)$.

Proof. The fact that $N_{1}(S(X, G))=F\left(Y_{d}, G\right)$ is just Theorem 2.5 of [15]. We know that $\left(Y_{d}, \nu, G\right)=\left(G / G_{0}, \nu, G\right)$ for some subgroup $G_{0}$ of finite index. If $\left(Z_{d}, \tau_{d}, G\right)$ is the maximal quotient action of $(X, \mu, G)$ having relatively discrete spectrum over $\left(Y_{d}, \nu, G\right)$, Zimmer's structure theory shows us that $\left(Z_{d}, \tau_{d}, G\right)=\left(Y_{d} \times{ }_{\alpha} K / H, \nu \times \nu_{K / H}, G\right)$, where $\alpha$ : $Y_{d} \times G \rightarrow K$ is a one cocycle with $\alpha$ minimal and $K_{\alpha}=K$. It is an easy exercise using the transitivity of $\left(Y_{d}, \nu, G\right)$ and [26, Theorem 8.27] to show that we may take $H=\{e\}, K$ abelian, and then $\left(Y \times_{\alpha} K, \nu \times \nu_{K}, G\right)$ has pure point spectrum. Since $\left(Y_{d}, \nu, G\right)$ is the maximal quotient action having pure point spectrum $K=\{e\}$, thus $(X, \mu, G)$ is relatively weak mixing over $\left(Y_{d}, \nu, G\right)$, hence $N_{1}\left(F\left(Y_{d}, G\right)\right)=F\left(Y_{d}, G\right)$ by the theorem.

REMARK 3.6. Since any ergodic action of $\mathbf{Z}$ having pure point spectrum is either free or transitive, Corollary 3.5 allows us to dispense with the hypothesis of freeness of the maximal action with pure point spectrum in Corollary 2.9 when $G=\mathbf{Z}$. Hence we can always compute the length of $S(X, \mathbf{Z})$ in $F(X, \mathbf{Z})$ when given a free ergodic action $\mathbf{Z}$ on $(X, \mu)$ which preserves the finite measure $\mu$.

4. Compact skew product extensions of ergodic actions and the corresponding crossed dual product von Neumann algebras. Suppose that $K$ is a compact (second countable) group and $\alpha: Y \times G \rightarrow K$ is a one-cocycle, where $(Y, \nu, G)$ is an ergodic $G$-space. When $K$ is abelian, there is a spatial automorphism between $F\left(Y \times{ }_{\alpha} K, G\right)$ and a crossed product $F(Y, G) \times_{\hat{\alpha}} \hat{K}$ which carries $\varphi^{*}(F(Y, G))$ onto $F(Y, G) \times_{\hat{\alpha}}\{e\}$, where $\varphi: Y \times K \rightarrow Y$ is projection in the first variable. This situation was exploited in [15], and indeed the main result of the second section of this 
paper shows that the normalizer of a subfactor corresponding to a quotient action is equal to the crossed product of the subfactor by a particular countable abelian group. The main aim of this section will be to examine the structure of the factors $F\left(Y \times{ }_{\alpha} K, G\right)$ where $K$ is a compact not necessarily abelian group. If $K$ is no longer abelian the idea of a crossed product of $F(Y, G)$ by an action of " $\hat{K}$ " no longer makes sense. However, a substitute construction, the "crossed-dual product," does exist in the literature; it reduces to the regular crossed product by $\hat{K}$ when $K$ is abelian. This construction was developed almost simultaneously in [11], [7] and [20] in order to extend Takesaki duality to non-abelian locally compact groups. In what follows the version of the crossed dual product due to Nakagami will be used [10]. The main result of this section states that if $(X, \mu, G)$ is an ergodic extension of the free ergodic action $(Y, \nu, G)$ with $\mu$ relatively $G$-invariant over $\nu$, then $F(X, G)$ is spatially isomorphic to a crossed dual product of $F(Y, G)$ by a compact (second countable) group $K$ with suitable embedding conditions if and only if $(X, \mu, G)$ is essentially isomorphic to $\left(Y \times_{\alpha} K, \nu \times \nu_{K}, G\right)$ for some one cocycle $\alpha$ : $Y \times G \rightarrow K$ with dense range. In what follows all groups considered will be second-countable.

We recall the relevant definitions:

Definition 4.1 [11]. Let $\mathscr{M}$ be a von Neumann algebra, acting on the separable Hilbert space $\mathscr{H}$, and let $K$ be an arbitrary locally compact group. An abstract co-action $\beta$ of $K$ on $\mathscr{M}$ is a von Neumann algebra monomorphism

$$
\beta: \mathscr{M} \rightarrow \mathscr{M} \otimes \mathscr{R}(K)
$$

where $\mathscr{R}(K)$ is the von Neumann algebra generated by the right regular representation of $K$ on $L^{2}(K)$, which satisfies the following commutative diagram:

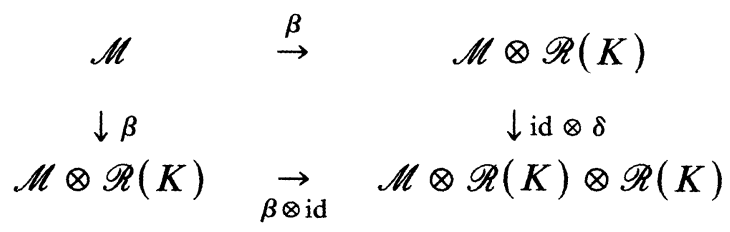

Here $\delta: \mathscr{R}(K) \rightarrow \mathscr{R}(K) \otimes \mathscr{R}(K)$ is the map defined by $\delta\left(\rho_{k_{1}}\right)=\rho_{k_{1}} \otimes \rho_{k_{1}}$, where $\rho_{k_{1}}(f) k=f\left(k k_{1}\right), k, k_{1} \in K$.

The crossed dual product of $\mathscr{M}$ by the abstract coaction $\beta$ of $K$ is the von Neumann algebra acting on $\mathscr{H} \otimes L^{2}(K)$ generated by $\beta(\mathscr{M})$ and $C_{\mathscr{H}} \otimes L^{\infty}(K)$. Denote this von Neumann algebra by $\mathscr{M} \times{ }_{\beta}^{d} K$. 
REMARK 4.2. The reader is referred to [11], [12], for an abstract definition of action of a locally compact second countable group $K$ on a von Neumann algebra very similar to that given above for coactions. Using such a definition, one can show that whenever $K$ is abelian, an action of $\hat{K}$ on $\mathscr{M}$ defines a coaction of $K$ on $\mathscr{M}$ (and conversely), with $\mathscr{M} \times \hat{K}$ spatially $*$-isomorphic to $\mathscr{M} \times{ }^{d} K$.

Given a coaction $\beta$ of the unimodular locally compact group $K$ on $\mathscr{M}$, one defines an action of $K$ on $\mathscr{M} \times{ }_{\beta}^{d} K$ as follows.

Let $\ell(k): L^{2}(K) \rightarrow L^{2}(K)$ be defined as

$$
\ell\left(k_{1}\right) f(k)=f\left(k_{1}^{-1} k\right) \text {. }
$$

Then $\forall k \in K, \operatorname{Id}_{\mathscr{H}} \otimes \ell(k)$ is a unitary in $\mathscr{B}\left(\mathscr{H} \otimes L^{2}(K)\right)$, and $\operatorname{Ad}\left(\operatorname{Id}_{\mathscr{H}} \otimes\right.$ $\ell(k))$ acts as the identity on $\mathscr{M} \otimes \mathscr{R}(K)$ and leaves $\mathbf{C} \otimes L^{\infty}(K)$ invariant. Thus $\operatorname{Ad}\left(\operatorname{Id}_{\mathscr{H}} \otimes \ell(K)\right)$ fixes $\left(\mathscr{M} \times{ }_{\beta}^{d} K\right)$. Let $\hat{\beta}(k)(m)=\operatorname{Ad}\left(\operatorname{Id}_{\mathscr{H}}\right.$ $\otimes \ell(k))(m), \forall m \in \mathscr{M} \times{ }_{\beta}^{d} K, \forall k \in K$. Then $\hat{\beta}$ defines an action of $K$ on $\mathscr{M} \times{ }_{\beta}^{d} K$, called the action dual to the coaction $\beta$. When $K$ is abelian $\hat{\beta}$ corresponds in a natural way to Takesaki's dual action of $K$ on $\mathscr{M} \times \hat{K}$. Results from Takesaki-Nagakami duality theory [11], [12] show that the von Neumann subalgebra of $\mathscr{M} \times{ }_{\beta}^{d} K$ consisting of elements left fixed by the action $\hat{\beta}$ of $K$ is precisely $\beta(\mathscr{M})$. Furthermore the crossed product of $\mathscr{M} \times{ }_{\beta}^{d} K$ by the dual action $\hat{\beta}$ of $K$ is $*$-isomorphic to $\mathscr{M} \otimes \mathscr{B}\left(L^{2}(K)\right)$.

Let $K$ be a compact group and let $(Y, \nu, G)$ be an ergodic $G$-space. For any one cocycle $\alpha: Y \times G \rightarrow K$ form the skew product action $\left(Y \times{ }_{\alpha} K, \nu \times \nu_{K}, G\right)$; note that $\nu \times \nu_{K}$ is relatively $G$-invariant over $\nu=\varphi_{*}\left(\nu \times \nu_{K}\right)$ where $\varphi: Y \times_{\alpha} K \rightarrow Y$ is given by $\varphi((y, k))=y$. From what has been said previously one expects that $F\left(Y \times{ }_{\alpha} K, G\right)$ has the structure of a crossed dual product of $F(Y, G)$ by a coaction of $K$, and this is in fact the case.

THEOREM 4.3. Let $(Y, \nu, G)$ be an ergodic G-space, $\alpha: Y \times G \rightarrow K a$ one-cocycle, and $\varphi: Y \times_{\alpha} K \rightarrow Y$ the quotient $G$ map of $\left(Y \times{ }_{\alpha} K, \nu \times \nu_{K}\right)$ onto $(Y, \nu)$ given by projection in the first variable. Then $\varphi^{*}: F(Y, G) \rightarrow$ $F\left(Y \times{ }_{\alpha} K, G\right)$ defines a coaction of $K$ on $F(Y, G)$, and $F\left(Y \times{ }_{\alpha} K, G\right)=$ $F(Y, G) \times{ }_{\varphi^{*}}^{d} K$. Furthermore the dual action $\hat{\varphi}^{*}$ of $K$ on $F\left(Y \times{ }_{\alpha} K, G\right)$ is defined by

$$
\hat{\varphi}^{*}(k)\left(\sum_{g \in G} T_{f_{g}} U_{g}\right)=\sum T_{\varepsilon(k) f_{g}} U_{g}, \quad \text { where } \varepsilon\left(k_{1}\right) f(y, k)=f\left(y, k_{1}^{-1} k\right) \text {. }
$$


Proof. In what follows we use the natural identification of $L^{2}(Y) \otimes$ $L^{2}(G) \otimes L^{2}(K)$ with $L^{2}(Y \times K \times G)$ defined by

$$
u_{1}(y) u_{2}(g) u_{3}(k) \mapsto u_{1}(y) \otimes u_{2}(g) \otimes u_{3}(k) .
$$

We first show that the image of $\varphi^{*}$ lies in $F(Y, G) \otimes \mathscr{R}(K)$. Recall that $F(Y, G)$ contains the subalgebra $R(Y, G)$ which can be identified with $L^{\infty}(Y, \nu)$ and is, in fact, represented on $L^{2}(Y \times G)=L^{2}(Y) \otimes L^{2}(G)$ as $L^{\infty}(Y, \nu) \otimes$ C. Therefore the von Neumann algebra $F(Y, G) \otimes \mathscr{R}(K)$ contains as a von Neumann subalgebra $L^{\infty}(Y) \otimes \mathscr{R}(K)$. This last can be naturally identified with $L^{\infty}(Y, \mathscr{R}(K))$, the bounded Borel fields of operators $(y \mapsto b(y))$ with each $b(y) \in \mathscr{R}(K)$. We now claim that for every $g_{1} \in G, \varphi^{*}\left(U_{g_{1}}^{Y}\right)=U_{g_{1}}^{X}$ can be decomposed

$$
\varphi^{*}\left(U_{g_{1}}^{Y}\right)=\left(y \mapsto \rho\left(\alpha\left(y, g_{1}\right)\right)\right)\left(U_{g_{1}}^{Y} \otimes \operatorname{Id}_{L^{2}(K)}\right) .
$$

Let $u_{1} \in L^{2}(Y), u_{2} \in L^{2}(G), u_{3} \in L^{2}(K)$. Then

$$
\begin{aligned}
(y \mapsto & \left.\rho\left(\alpha\left(y, g_{1}\right)\right)\right)\left(U_{g_{1}}^{Y} \otimes \mathbf{I d}\right)\left(u_{1} \otimes u_{2} \otimes u_{3}\right)(y, g, k) \\
& =\left(y \mapsto \rho\left(\alpha\left(y, g_{1}\right)\right)\right) r_{Y}\left(y, g_{1}\right)^{1 / 2} u_{1}\left(y g_{1}\right) \otimes u_{2}\left(g_{1}^{-1} g\right) \otimes u_{3}(k) \\
& =r_{Y}\left(y, g_{1}\right)^{1 / 2} u_{1}\left(y g_{1}\right) \otimes u_{2}\left(g_{1}^{-1} g\right) \otimes u_{3}\left(k \alpha\left(y, g_{1}\right)\right) .
\end{aligned}
$$

Setting $u(y, k, g)=u_{1}(y) u_{3}(k) u_{2}(g)$, then

$$
\begin{aligned}
\varphi^{*}\left(U_{g_{1}}^{Y}\right)(u)(y, k, g) & =r_{Y}\left(y, g_{1}\right)^{1 / 2} u\left((y, k) g_{1}, g_{1}^{-1} g\right) \\
& =r_{Y}\left(y, g_{1}\right)^{1 / 2} u\left(y g_{1}, k \alpha\left(y, g_{1}\right), g_{1}^{-1} g\right) \\
& =r_{Y}\left(y, g_{1}\right)^{1 / 2} u_{1}\left(y g_{1}\right) u_{3}\left(k \alpha\left(y, g_{1}\right)\right) u_{2}\left(g^{-1}, g\right) .
\end{aligned}
$$

Hence $\varphi^{*}\left(U_{g_{1}}^{Y}\right)=\left(y \mapsto \rho\left(\alpha\left(y, g_{1}\right)\right)\right)\left(U_{g_{1}}^{Y} \otimes \mathrm{Id}\right)$, which is contained in $F(Y, G) \otimes \mathscr{R}(K)$. Let $f$ be an arbitrary element of $L^{\infty}(Y)$. The $T_{f} \in$ $R(Y, G)$, and it is clear that $\varphi^{*}\left(T_{f}\right)=T_{f} \otimes \operatorname{Id}$ on $L^{2}(Y) \otimes L^{2}(G) \otimes L^{2}(K)$. Since $F(Y, G)$ is generated by $R(Y, G)$ and $\left\{U_{g} \mid g \in G\right\}$, and since $\varphi^{*}$ is a von Neumann algebra monomorphism, the image of $\varphi^{*}$ will lie in $F(Y, G) \otimes \mathscr{R}(K)$, with

$$
\varphi^{*}\left(\sum_{g \in G} T_{f_{g}} U_{g}^{Y}\right)=\sum_{g \in G}\left(y \rightarrow f_{g}(y) \rho(\alpha(y, g))\right)\left(U_{g}^{Y} \otimes \mathrm{Id}\right)
$$

for finite sums, and where if $\left\{\left.\sum_{g \in G} T_{f_{g}^{\alpha}} U_{g}\right|_{\alpha \in A}\right\}$ is a net of finite sums converging to $m \in F(Y, G)$ in the strong operator topology, then

$$
\left\{\varphi^{*}\left(\sum_{g \in G} T_{f_{g}^{\alpha}} U_{g}^{Y}\right)=\left.\sum_{g \in G}\left(y \rightarrow f_{g}^{\alpha}(g) \rho(\alpha(y, g))\right)\left(U_{g}^{Y} \otimes \mathrm{Id}\right)\right|_{\alpha \in A}\right\}
$$


is a net in $F(Y, G) \otimes \mathscr{R}(K)$ converging to $\varphi^{*}(m)$ in the strong operator topology. We now check that $\varphi^{*} \otimes$ id $\circ \varphi^{*}=($ id $\otimes \delta) \varphi^{*}$. Recall that there is a natural identification of $L^{\infty}(Y, \mathscr{R}(K)) \otimes \mathscr{R}(K)$ with $L^{\infty}(Y, \mathscr{R}(K) \otimes \mathscr{R}(K))$. Then on finite sums,

$$
\begin{aligned}
\left(\varphi^{*} \otimes \mathrm{Id}\right) & \varphi^{*}\left(\sum_{g \in G} T_{f_{g}} U_{g}^{Y}\right) \\
= & \varphi^{*} \otimes \mathrm{Id}\left(\sum_{g \in G}\left(y \mapsto f_{g}(y) \rho(\alpha(y, g))\right)\left(U_{g}^{Y} \otimes \mathrm{Id}\right)\right) \\
= & \sum_{g \in G}\left[\left(y \mapsto \mathrm{Id} \otimes f_{g}(y) \rho(\alpha(y, g))\right)(y \mapsto \rho(\alpha(y, g)) \otimes \mathrm{Id})\right. \\
= & \sum_{g \in G}\left[\left(y \mapsto f_{g}(y) \rho(\alpha(y, g)) \otimes \rho(\alpha(y, g))\right)\left(U_{g}^{Y} \otimes \mathrm{Id} \otimes \mathrm{Id}\right)\right] \\
= & \operatorname{Id} \otimes \delta\left(\sum_{g \in G}\left[\left(y \mapsto f_{g}(y) \rho(\alpha(y, g))\right)\left(U_{g}^{Y} \otimes \mathrm{Id}\right)\right]\right) \\
= & (\operatorname{Id} \otimes \delta) \varphi^{*}\left(\sum_{g \in G} T_{f_{g}} U_{g}\right) .
\end{aligned}
$$

By continuity arguments, $\left(\varphi^{*} \otimes \mathrm{id}\right) \circ \varphi^{*}=(\mathrm{id} \otimes \delta) \circ \varphi^{*}$ on all of $F(Y, G)$, so that $\varphi^{*}$ defines a coaction of $K$ on $F(Y, G)$ as desired.

Let $\psi: Y \times K \rightarrow K$ be defined by $\psi(y, k)=k$. Then if $\gamma \in L^{\infty}(K)$, $\gamma \circ \psi \in L^{\infty}(Y \times K)$. Examine $T_{\gamma \circ \psi} \in F\left(Y \times{ }_{\alpha} K, G\right)$ :

Therefore

$$
\begin{aligned}
T_{\gamma \circ \psi}\left(u_{1} u_{2} u_{3}\right)(y, g, k) & =\gamma(k) u_{1}(y) u_{2}(g) u_{3}(k) \\
& =u_{1}(y) u_{2}(g) \gamma(k) u_{3}(k) .
\end{aligned}
$$

$$
T_{\gamma \circ \psi}=\operatorname{Id}_{L^{2}(Y \times G)} \otimes \gamma .
$$

Since $\varphi^{*}(F(Y, G))$ and $\left\{T_{\gamma \circ \psi} \mid \gamma \in L^{\infty}(K)\right\}$ generate $F\left(Y \times_{\alpha} K, G\right)$, we see that $F(Y, G) \times_{\varphi^{*}}^{d} K$ is precisely $F\left(Y \times{ }_{\alpha} K, G\right)$.

Finally, note that for $f \in L^{\infty}(Y \times K), g_{1} \in G, K_{1} \in K$ Id $\otimes \ell\left(k_{1}\right)\left(T_{f} U_{g_{1}}\right) \operatorname{Id} \otimes \ell\left(k_{1}^{-1}\right) u_{1} u_{2} u_{3}(y, g, k)$

$$
\begin{aligned}
& =\mathrm{Id} \otimes \ell\left(k_{1}\right) T_{f} U_{g_{1}} u_{1}(y) u_{2}(g) u_{3}\left(k_{1} k\right) \\
& =\mathrm{Id} \otimes \ell\left(k_{1}\right) r_{Y}\left(y, g_{1}\right)^{1 / 2} f(y, k) u_{1}\left(y g_{1}\right) u_{2}\left(g_{1}^{-1} g\right) u_{3}\left(k_{1} k \alpha\left(y, g_{1}\right)\right) \\
& =r_{Y}\left(y, g_{1}\right)^{1 / 2} f\left(y, k_{1}^{-1} k\right) u_{1}(y g) u_{2}\left(g_{1}^{-1} g\right) u_{3}\left(k \alpha\left(y, g_{1}\right)\right) \\
& =T_{\varepsilon\left(k_{1}\right) f} U_{g_{1}}\left(u_{1} u_{2} u_{3}\right)(y, g, k) .
\end{aligned}
$$


Since $\operatorname{Ad}\left(\operatorname{Id} \otimes \ell\left(k_{1}\right)\right)$ is continuous on $\mathscr{B}\left(L^{2}(Y \times G \times K)\right)$,

$$
\operatorname{Ad}\left(\operatorname{Id} \otimes \ell\left(k_{1}\right)\right)\left(\sum_{g \in G} T_{f_{g}} U_{g}\right)=\hat{\varphi}^{*}\left(k_{1}\right)\left(\sum_{g \in G} T_{f_{g}} U_{g}\right)=\sum_{g \in G} T_{\varepsilon\left(k_{1}\right) f_{g}} U_{g} .
$$

This completes the proof.

REMARK 4.4. Recall that an automorphism of groupoids $\Lambda: \mathscr{G} \rightarrow \mathscr{G}$ gives rise to an automorphism of von Neumann algebras $\Lambda^{*}: L(\mathscr{G}) \rightarrow$ $L(\mathscr{G})$, where $L(\mathscr{G})$ is the von Neumann algebra corresponding to the left regular representation of $\mathscr{G}$ [6]. Then an easy computation shows that the *-isomorphism $\hat{\varphi}^{*}\left(k_{1}\right)$ corresponds to the automorphism of the groupoid $Y \times{ }_{\alpha} K \times G$ defined by $(y, k, g) \mapsto\left(y, k_{1} k, g\right)$.

Let $(Y, \nu, G)$ be a free, properly ergodic action of the discrete abelian group $G$ which preserves the measure $\nu$. For any separable compact group $K$, Zimmer has shown [29] that there exists a one cocycle $\alpha: Y \times G \rightarrow K$ with dense range, so that the skew product action $\left(Y \times_{\alpha} K, \nu \times \nu_{K}, G\right)$ is ergodic. Since $G$ is abelian and preserves the measure $\nu \times \nu_{K}, F\left(Y \times{ }_{\alpha} K\right.$, $G$ ) is the hyperfinite $\mathrm{II}_{1}$ factor. (Results of Pasche [16] also imply this fact.) Let $\beta$ be the coaction of $K$ on $F(Y, G)$ given by $\varphi^{*}$. Then $F\left(Y \times{ }_{\alpha} K\right.$, $G) \times_{\hat{\beta}} K$ is *-isomorphic to $F(Y, G) \otimes \mathscr{B}\left(L^{2}(K)\right)$ by Nakagami-Takesaki duality, and thus is a factor. This combination of Zimmer's and Nakagami's results shows the following, first proved in [16]:

THEOREM 4.5. For any compact second countable group $K$, there exists an outer action of $K$ on the hyperfinite $\mathrm{II}_{1}$ factor $\mathscr{R}$ such that the crossed product of $\mathscr{R}$ by this action is again a factor.

Proof. All that remains to be shown is that the action $\hat{\beta}$ discussed above is an outer action. Suppose that there exists a $k \in K$ and $U \in$ $F\left(Y \times_{\alpha} K, G\right)$ such that

$$
\hat{\beta}(k)=\operatorname{Ad} U .
$$

We note that $\hat{\beta}(k)$ leaves everything in $\varphi^{*}(R(Y, G))$ fixed. Hence by Lemma 1.6, $U=T_{f}$ for some $f \in L^{\infty}(X)$. But this implies that $\hat{\beta}(k)$ leaves everything in $R(X, G)$ fixed, hence every element of $F(X, G)$ fixed. Therefore $\hat{\beta}(k)$ is the identity automorphism, which shows that $k=e$, as desired.

A natural question that arises in our context is that of when is $F(X, G) *$-isomorphic to a crossed dual product of $F(Y, G)$, for $(Y, \nu, G)$ 
a quotient action of $(X, \mu, G)$. The following theorem provides a partial converse to Theorem 4.4:

TheOREM 4.6. Let $(X, \mu, G)$ be an ergodic $G$-space, and let $(Y, \nu, G)$ be a free quotient action, with quotient map $\varphi: X \rightarrow Y$, where $\mu$ is relatively $G$-invariant over $\nu=\varphi_{*} \mu$. Then $F(X, G)$ is spatially *-isomorphic to a crossed dual product of $F(Y, G)$ by coaction $\beta$ of a compact group $K$, and under this *-isomorphism $\varphi^{*}(F(Y, G))$ is carried onto $\beta(F(Y, G))$, if and only if $(X, \mu, G)$ is essentially isomorphic as an extension to $\left(Y \times{ }_{\alpha} K\right.$, $\left.\nu \times \nu_{K}, G\right)$ for some one cocycle $\alpha: Y \times G \rightarrow K$ with dense range.

Proof. The direction $\Leftarrow$ was proved in Theorem 4.3 As for $\Rightarrow$, suppose that $F(X, G)$ is spatially *-isomorphic to a crossed dual product of $F(Y, G)$ by some coaction $\beta$ of a compact (second countable) group $K$; then there exists a dual action $\hat{\beta}$ of $K$ on $F(X, G)$ leaving every element of $\beta(F(Y, G))=\varphi^{*}(F(Y, G))$ fixed. By the results of $\S 1, \varphi^{*}(R(Y, G))$ is contained in a unique maximal abelian subalgebra of $F(X, G)$, namely $R(X, G)$. Therefore for every $k \in K, \hat{\beta}(k)$ leaves $R(X, G)$ invariant, since $\hat{\beta}$ leaves every element of $\varphi^{*}(R(Y, G))$ fixed and $\hat{\beta}(k)$ is a $*$-automorphism. Thus the action of $\beta$ of $K$ on $F(X, G)$ restricts to give an action of $K$ on $R(X, G) \cong L^{\infty}(X)$. Furthermore, for every $k \in K$, the $*$-automorphism $\hat{\beta}(k)$ commutes with the action of $G$ on $R(X, G)$ induced by the adjoint action of $\left\{U_{g} \mid g \in G\right\}$ (which corresponds to the action of $G$ on $(X, \mu)$ ). Indeed $\forall g \in G, \forall k \in K, \forall f \in L^{\infty}(X)$,

$$
\begin{aligned}
\hat{\beta}(k)\left(U_{g} T_{f} U_{g}^{*}\right) & =\hat{\beta}(k)\left(U_{g}\right) \hat{\beta}(k)\left(T_{f}\right) \hat{\beta}(k)\left(U_{g}^{*}\right) \\
& =U_{g}\left(\hat{\beta}(k)\left(T_{f}\right)\right) U_{g}^{*},
\end{aligned}
$$

since $U_{g} \in \varphi^{*}(F(Y, G))=\beta(F(Y, G))$.

One therefore obtains an action of the product group $G \times K$ on $R(X, G) \cong L^{\infty}(X)$ defined by

$$
\left(T_{f}\right) \cdot(g, k)=U_{g} \hat{\beta}(k)\left(T_{f}\right) U_{g}^{*} .
$$

By a result of Mackey [8] we obtain a point action of $G \times K$ on $(X, \mu)$ leaving the measure $\mu$ quasi-invariant. This action must be ergodic, because the action restricted to the subgroup $G \times\{e\}$ is ergodic. By a result of Series [18], there exists an ergodic $G$ space $\left(S_{1}, \mu\right)$, a transitive $K$-space $\left(S_{2}, \mu_{2}\right)$, a locally compact group $\Gamma$, and one cocycles $\Pi_{1}, \Pi_{2}$, $\Pi_{1}: S_{1} \times G \rightarrow \Gamma, \Pi_{2}: S_{2} \times K \rightarrow \Gamma$, such that $(X, \mu, G \times K)$ is measuretheoretically conjugate to the $G \times K$ space $\left(S_{1} \times S_{2} \times \Gamma, \mu_{1} \times \mu_{2} \times \nu_{\Gamma}\right)$ where $\left(s_{1}, s_{2}, \gamma\right)(g, k)=\left(s_{1} g, s_{2} k, \Pi_{2}\left(s_{2}, k\right)^{-1} \gamma \Pi_{1}\left(s_{1}, g\right)\right)$. Furthermore, 
$\left(S_{i}, \mu_{i}\right), i=1,2$, can be chosen so that $\left(S_{1}, \mu_{1}\right)$ is the quotient of $(X, \mu)$ by the $\{e\} \times K$ action and $\left(S_{2}, \mu_{2}\right)$ is the quotient of $(X, \mu)$ by the $G \times\{e\}$ action [18]. Since $G \times\{e\}$ acts ergodically on $(X, \mu),\left(S_{2}, \mu_{2}\right)=$ pt., and $\Pi_{1}: S_{1} \times G \rightarrow \Gamma$ must have dense range. It follows that $\Pi_{2}: S_{2} \times K \rightarrow \Gamma$ is in fact a homomorphism of $K$ into $\Gamma$. Also, $\Gamma$ is isomorphic to $K / L$, where $L$ is a normal subgroup of $K$ corresponding to the stability subgroup for the action of $K$ on $(X, \mu)$ at some generic point ([18] Remark 5.1). We claim $\left(S_{1}, \mu_{1}, G\right)$ is conjugate to $(Y, \nu, G)$ and $L=\{e\}$. For the first assertion, note that the fixed points for the action of $K$ on $R(X, G) \cong$ $L^{\infty}(X)$ are precisely $\varphi^{*}(R(Y, G))=L^{\infty}(Y, \nu)$. Hence $(Y, \nu)$ is the quotient of the $K$ action on $(X, \mu)$ and thus $(Y, \nu, G)$ is conjugate to $\left(S_{1}, \mu_{1}, G\right)$. As for the second assertion, note that for every $\ell \in L, \hat{\beta}(\ell)$ leaves every element of $F(X, G)$ fixed. This is clear since $F(X, G)=$ $F\left(Y \times_{\mathrm{II}_{1}} K / L, G\right)$ and $\hat{\beta}(k)$ acts by translation on $K / L$ alone. But functions on $K / L$ remain fixed under translation by elements of $L$. Since by hypothesis $F(X, G)$ is spatially *-isomorphic to the crossed dual product of $F(Y, G)$ by a coaction on $K, F(X, G)$ must contain a copy of the abelian subalgebra $\mathbf{C} \otimes L^{\infty}(K)$, where the action $\hat{\beta}$ on $K$ on this subalgebra is given by the left regular representation. Clearly the kernel of the action of $\hat{\beta}$ restricted to $\mathbf{C} \otimes L^{\infty}(K)$ is the identity element of $K$. But this kernel must contain $L$, since $\{\hat{\beta}(\ell) \mid \ell \in L\}$ leaves everything in $F(X, G)$ fixed. Therefore $L=\{e\}$, and the desired result is obtained with $\alpha=$ $\Pi_{1}$.

Relationship to normal extensions.

We recall the definition of normal extensions due to Zimmer [27]:

Definition 4.7. Let $(X, \mu, G)$ be an ergodic $G$-space, and suppose that $(Y, \nu, G)$ is a quotient $G$-space, with $\varphi: X \rightarrow Y$, and the measure $\mu$ relatively $G$-invariant over $\nu=\varphi_{*} \mu$. For every $y \in Y$ and for every $x \in \varphi^{-1}(y)$, let $\mathscr{H}_{x}=L^{2}\left(\varphi^{-1}(y), \mu_{y}\right)$. Let $\alpha(y, g): \mathscr{H}_{y g} \rightarrow \mathscr{H}_{y}$ be the natural bundle cocycle representation, and restrict this representation to $X \times G$ by defining $\alpha(\varphi(x), g): \mathscr{H}_{x g} \mapsto \mathscr{H}_{x}$. Then $(X, \mu, G)$ is said to be a normal extension of $(Y, \nu, G)$ if there exists a spearable Hilbert space $\mathscr{H}_{0}$ and a Borel field of unitary operators $U(x): \mathscr{H}_{x} \rightarrow \mathscr{H}_{0}$ with

$$
U(x) \alpha(\varphi(x), g) U(x g)^{-1}=\operatorname{Id}_{\mathscr{H}_{0}} .
$$

By results of Zimmer [27], extended to the case $\mu$ relatively $G$-invariant over $\nu$ by Fabec [3], if $\mu$ is finite and if $(X, \mu, G)$ is a normal ergodic extension of $(Y, \nu, G)$ having relatively discrete spectrum over 
$(Y, \nu, G)$ then $(X, \mu, G)$ is essentially isomorphic as an extension to $\left(Y \times{ }_{\alpha} K, \nu \times \nu_{K}, G\right)$, where $\alpha: Y \times G \rightarrow K$ is a one-cocycle with dense range. We can thus rephrase Theorem 4.6 as

COROllary 4.8. Let $(X, \mu, G)$ be an ergodic action of the countable group $G$ on the compact Lebesgue space $(X, \mu)$, and let $(Y, \nu, G)$ be a free quotient action, with $\varphi: Y \rightarrow Y$, such that $\mu$ is relatively G-invariant over $\nu=\varphi_{*} \mu$. Then $F(X, G)$ is spatially *-isomorphic to the crossed dual product of $F(Y, G)$ by a coaction $\beta$ of some compact group and this *-isomorphism carries $\varphi^{*}(F(Y, G))$ onto $\beta(F(Y, G))$ if and only if $(X, \mu, G)$ is a normal extension of $(Y, \nu, G)$ with relatively discrete spectrum over $(Y, \nu, G)$.

REMARK 4.9. The results in ([27], Theorem 6.2) show that the compact group of Corollary 4.8 must be unique up to isomorphism.

Intermediate subalgebras.

Notation 4.10. Let $\mathscr{M}$ be a von Neumann algebra acting on the separable Hilbert space $\mathscr{H}$ and suppose that $\beta$ is a coaction of the unimodular locally compact group $K$ on $\mathscr{M}$. Let $H$ be a closed subgroup of $K$. Then as in [12] set

$$
\mathscr{M} x_{\beta}^{d} H / K=\left\{m \in \mathscr{M} \times{ }_{\beta}^{d} K \mid \hat{\beta}(h)(m)=m \forall h \in H\right\} .
$$

It follows that $\mathscr{M} \times{ }_{\beta}^{d} H / K$ is a von Neumann subalgebra of $\mathscr{M} \times{ }_{\beta}^{d} K$ with

$$
\beta(\mathscr{M}) \subset \mathscr{M} \times{ }_{\beta}^{d} H / K \subset \mathscr{M} \times{ }_{\beta}^{d} K .
$$

Hence closed subgroups of $K$ give rise to intermediate subalgebras in the crossed dual product construction. In our context the $\mathscr{M} \times{ }_{\beta}^{d} H / K$ take on a natural form corresponding to quotient actions: recall that if $K$ is a compact group and $\alpha: Y \times G \rightarrow K$ is a one-cocycle, where $(Y, \nu, G)$ is an ergodic action, then the dual action of $\hat{\varphi}^{*}$ of $K$ on $F\left(Y \times_{\alpha} K, G\right)$ is given by

$$
\hat{\varphi}^{*}(k)\left(\sum_{g \in G} T_{f_{g}} U_{g}\right)=\sum_{g \in G} T_{\varepsilon_{k}\left(f_{g}\right)} U_{g}, \quad g \in G, f_{g} \in L^{\infty}(X)
$$

where $\varepsilon_{k_{1}}\left(f_{g}\right)(x, k)=f_{g}\left(x, k_{1}^{-1} k\right)$. If $H$ is closed subgroup of $K$, it is clear that the fixed points of $\left\{\hat{\beta}_{\alpha}(h) \mid h \in H\right\}$ in $F\left(Y \times_{\alpha} K, G\right)$ are given by

$$
\left\{\sum_{g \in G} T_{f_{g}} U_{g} \in F\left(Y \times{ }_{\alpha} K, G\right) \mid \varepsilon_{h}\left(f_{g}\right)=f_{g} \forall h \in H, \forall g \in G\right\} .
$$


But $L^{\infty}$ function on $\left(Y \times_{\alpha} K, \nu \times \nu_{K}\right)$ invariant under $\{\varepsilon(h) \mid h \in H\}$ are precisely those which are lifts of functions on $\left(Y \times K / H, \nu \times \nu_{K / H}\right)$ Thus letting $\varphi_{H}:\left(Y \times{ }_{\alpha} K, \nu \times \nu_{K}\right) \rightarrow\left(Y \times K / H, \nu \times \nu_{K / H}\right)$ be defined by $\varphi_{H}(y, k)=(y, \bar{k})$, we obtain a quotient map of $G$-spaces, and we have established that

$$
\varphi_{H}^{*}\left(F\left(Y \times{ }_{\alpha} K / H, G\right)\right)=F(Y, G) \times{ }^{d} H / K .
$$

In [12], a "Galois" correspondence is established between closed subgroups of $K$ and intermediate subalgebras of $\beta(\mathscr{M})$ and $\mathscr{M} \times{ }_{\beta}^{d} K$ satisfying certain embedding conditions when $\mathscr{M} \times{ }_{\beta}^{d} K$ is a factor. A combination of previous results in this paper allow us to show

THEOREM 4.11. Let $(X, \mu, G)$ be an ergodic $G$-space and $(Y, \nu, G)$ a free quotient $G$ space with $\varphi: X \rightarrow Y$, where $\mu$ is finite and relatively $G$-invariant over $\nu=\varphi_{*} \mu$. Suppose further that $(X, \mu, G)$ is a normal extension with relatively discrete spectrum over $(Y, \nu, G)$. Then there exists a compact second countable group $K$ unique up to isomorphism and a coaction $\beta$ of $K$ on $F(Y, G)$ such that $F(X, G)=F(Y, G) \times{ }_{\beta}^{d} K$ with $\varphi^{*}(F(Y, G))=$ $\beta(F(Y, G))$. Furthermore the intermediate von Neumann subalgebras between $F(Y, G)$ and $F(X, G)$ which are the image of a normal faithful conditional expectation are in one-to-one correspondence with closed subgroups $H$ of $K$. If $\mathcal{N}$ is such an intermediate von Neumann subalgebra, with associated subgroup $H_{\mathscr{N}}$, then $\mathscr{N}$ is invariant under the dual action $\hat{\beta}$ of $K$ on $F(X, G)$ if and only if $H_{\mathcal{N}}$ is normal in $K$.

Proof. The first sentence of the theorem is just a restatement of Theorem 4.4. Let $\mathcal{N}$ be an intermediate von Neumann subalgebra of $F(X, G)$ and $F(Y, G)$ which is the image of a normal faithful conditional expectation. By Theorem $1.9, \mathscr{N}=\varphi^{*}(F(Z, G))$ where $(Z, \tau, G)$ is an intermediate quotient action of $(X, \mu, G)$ and $(Y, \nu, G)$ with quotient $G$-map $\varphi:(X, \mu) \rightarrow(Z, \tau)$. Since $(X, \mu, G)$ is essentially isomorphic to $\left(Y \times{ }_{\alpha} K, \nu \times \nu_{K}, G\right)$ for some one-cocycle $\alpha: Y \times G \rightarrow K$ with dense range, $(Z, \tau, G)$ will look like $\left(Y \times{ }_{\alpha} K / H_{\mathcal{N}}, \nu \times \nu_{K / H}, G\right)$ for some closed subgroup $H_{\mathscr{N}}$ of $K$. Thus by our remarks preceding this theorem we see that

$$
\mathscr{N}=F(Y, G) \times{ }_{\beta}^{d} H_{\mathcal{N}} / K
$$

Finally, it is clear that $F(Y, G) \times{ }_{\beta}^{d} H_{\mathscr{N}} / K$ will be invariant under $\{\hat{\beta}(k) \mid k$ $\in K\}$ if and only if $H_{\mathcal{N}}$ is normal in $K$. This completes the proof. 


\section{REFERENCES}

[1] J. Dixmier, Sous-anneaux abéliens maximaux dans les facteurs de type fini, Ann. Math., 59 (1954), 279-296.

[2] Les Algebras d'Operateurs dans L'Espace Hibertien, Gauthier-Villars, Paris, 1957.

[3] R. Fabec, Normal ergodic actions and extensions, Israel J. Math., Vol. 40, No. 2, 175-186.

[4] , Cocycles, extensions of group actions, and bundle representations, J. Funct. Anal., 1984.

[5] P. Hahn, Haar measure for measure groupoids, Trans. Amer. Math. Soc., 242 (1978), $1-33$.

[6] The regular representation of measure groupoids, Trans. Amer. Math. Soc., 242 (1978), 35-72.

[7] M. Landstad, Duality theory for dual covariance algebras, Commun. Math. Phys., 52 (1977), 191-202.

[8] G. Mackey, Point realization of transformation groups, Illinois J. Math., 6 (1962), 327-335.

[9] _ Ergodic theory and virtual groups, Math. Annallen, 166 (1966), 187-207.

[10] C. C. Moore, Ergodicity of flows on homogeneous spaces, Amer. J. Math., 88 (1966), 154-178.

[11] Y. Nakagami, Dual action on a von Neumann algebra and Takesaki's duality for a locally compact group, Publ. R.I.M.S., Kyoto Univ., 12 (1977), 727-775.

[12] Y. Nakagami and M. Takesaki, Duality for Crossed Products of von Neumann Algebras, Springer Verlag, Berlin and New York, Lecture Notes \# 731.

[13] O. Nielsen, Maximal abelian subalgebras of hyperfinite factors, II, J. Funct. Anal., 6 (1970), 192-202.

[14] J. Packer, On the normalizer of certain subalgebras of group-measure factors, Bull. Amer. Math. Soc., (New Series) 7 (1982), 397-402.

[15] _ Point spectrum of ergodic abelian group actions and the corresponding groupmeasure factors, Pacific J. Math.

[16] W. Pasche, Inner product modules arising from compact automorphism groups of von Neumann algebras, Trans. Amer. Math. Soc., 224 (1976), 87-102.

[17] A. Ramsey, Virtual groups and group actions, Advances in Math., 6 (1971), 253-322.

[18] C. Series, Ergodic actions of product groups, Pacific J. Math., 70 (1977), 519-547.

[19] I. Singer, Automorphisms of finite factors, Amer. J. Math., 77 (1955), 117-133.

[20] S. Stratila, D. Voiculescu and L. Zsido, On crossed products, Rev. Roumaine Math. Pures et Appl., 21 (1976), 1411-1449.

[21] M. Takesaki, Conditional expectations in von Neumann algebras, J. Funct. Anal., 9 (1972), 306-321.

[22] Theory of Operator Algebras I, Springer-Verlag, Berlin and New York, 1979.

[23] R. J. Tauer, Maximal abelian subalgebras in finite factors of type $I_{1}$, Trans. Amer. Math. Soc., 114 (1965), 281-308.

[24] J. Tomiyama, On the projection of norm one in $W^{*}$-algebras, Proc. Japan Acad., 33 (1957), 608-612.

[25] H. Umegaki, Conditional expectation in an operator algebra I, Tôhoku Math. J., (1954), 177-181.

[26] V. Varadarajan, Geometry of Quantum Theory, Vol. II, Van Nostrand, Princeton, N. J., 1970.

[27] R. Zimmer, Extensions of ergodic actions, Illinois J. Math., 2 (1976), 373-409. 
[28] __ Ergodic actions with generalized discrete spectrum, Illinois J. Math., 20 (1976), 555-588.

[29] Cocycles and the structure of ergodic actions, Israel J. Math., 261 (1977), 214-220.

[30] Amenable ergodic group actions and an application to Poisson boundaries of random walks, J. Funct. Anal., 27 (1978), 350-372.

[31] Amenable pairs of groups and ergodic actions and the associated von Neumann algebras, Trans. Amer. Math. Soc., 243 (1978), 271-286.

Received April 4, 1983.

NATIONAL UNIVERSITY OF SINGAPORE

KENT Ridge, Singapore 0511 



\section{PACIFIC JOURNAL OF MATHEMATICS EDITORS}

\author{
V. S. VARADARAJAN (Managing Editor) \\ University of California \\ Los Angeles, CA 90024 \\ Charles R. DePrima \\ California Institute of Technology \\ Pasadena, CA 91125 \\ R. FInN \\ Stanford University \\ Stanford, CA 94305
}

\section{HeRmanN FlaschKa \\ University of Arizona \\ Tucson, AZ 85721}

Ramesh A. Gangolli

University of Washington

Seattle, WA 98195

ROBION KIRBY

University of California

Berkeley, CA 94720

\section{C. MOORE}

University of California

Berkeley, CA 94720

\section{H. SAMELSON}

Stanford University

Stanford, CA 94305

HAROLD STARK

University of California, San Diego

La Jolla, CA 92093

\section{ASSOCIATE EDITORS}
R. ARENS
E. F. BECKENBACH
B. H. NeumanN
F. Wolf
K. Yoshida (1906-1982)

\section{SUPPORTING INSTITUTIONS}
CALIFORNIA INSTITUTE OF TECHNOLOGY

\author{
UNIVERSITY OF SOUTHERN CALIFORNIA \\ STANFORD UNIVERSITY \\ UNIVERSITY OF HAWAII \\ UNIVERSITY OF TOKYO \\ UNIVERSITY OF UTAH \\ WASHINGTON STATE UNIVERSITY \\ UNIVERSITY OF WASHINGTON
}
UNIVERSITY OF OREGON

\begin{abstract}
UNIVERSITY OF ARIZONA
UNIVERSITY OF BRITISH COLUMBIA

UNIVERSITY OF CALIFORNIA

MONTANA STATE UNIVERSITY

UNIVERSITY OF NEVADA, RENO

NEW MEXICO STATE UNIVERSITY

OREGON STATE UNIVERSITY
\end{abstract}

The Supporting Institutions listed above contribute to the cost of publication of this Journal, but they are not owners or publishers and have no responsibility for its content or policies.

Mathematical papers intended for publication in the Pacific Journal of Mathematics should be in typed form or offset-reproduced (not dittoed), double spaced with large margins. Please do not use built up fractions in the text of the manuscript. However, you may use them in the displayed equations. Underline Greek letters in red, German in green, and script in blue. The first paragraph must be capable of being used separately as a synopsis of the entire paper. In particular it should contain no bibliographic references. Please propose a heading for the odd numbered pages of less than 35 characters. Manuscripts, in triplicate, may be sent to any one of the editors. Please classify according to the scheme of Math. Reviews, Index to Vol. 39. Supply name and address of author to whom proofs should be sent. All other communications should be addressed to the managing editor, or Elaine Barth, University of California, Los Angeles, California 90024.

There are page-charges associated with articles appearing in the Pacific Journal of Mathematics. These charges are expected to be paid by the author's University, Government Agency or Company. If the author or authors do not have access to such Institutional support these charges are waived. Single authors will receive 50 free reprints; joint authors will receive a total of 100 free reprints. Additional copies may be obtained at cost in multiples of 50 .

The Pacific Journal of Mathematics is issued monthly as of January 1966. Regular subscription rate: $\$ 190.00$ a year (5 Vols., 10 issues). Special rate: $\$ 66.00$ a year to individual members of supporting institutions.

Subscriptions, orders for numbers issued in the last three calendar years, and changes of address should be sent to Pacific Journal of Mathematics, P.O. Box 969, Carmel Valley, CA 93924, U.S.A. Old back numbers obtainable from Kraus Periodicals Co., Route 100, Millwood, NY 10546.

The Pacific Journal of Mathematics at P.O. Box 969, Carmel Valley, CA 93924 (ISSN 0030-8730) publishes 5 volumes per year. Application to mail at Second-class postage rates is pending at Carmel Valley, California, and additional mailing offices. Postmaster: Send address changes to Pacific Journal of Mathematics, P.O. Box 969, Carmel Valley, CA 93924.

PUBLISHED BY PACIFIC JOURNAL OF MATHEMATICS, A NON-PROFIT CORPORATION

Copyright $\odot 1985$ by Pacific Journal of Mathematics 


\section{Pacific Journal of Mathematics}

Vol. 119, No. $2 \quad$ June, 1985

Mustafa Agah Akcoglu and Meira Falkowitz (Soshniak), A general local

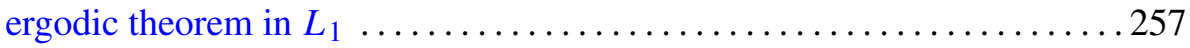

W. Wistar (William) Comfort and Lewis Chandlee Robertson,

Cardinality constraints for pseudocompact and for totally dense

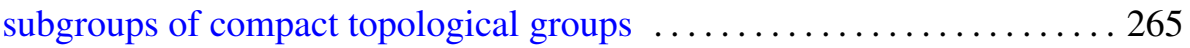

John Morse Delaurentis and Boris G. Pittel, Random permutations and

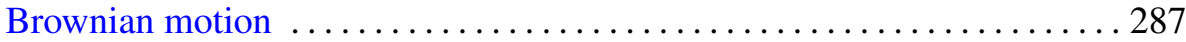

José Esteban Galé, Gel'fand theory in algebras of differentiable functions on Banach spaces ......................................... 303

Harry Gingold, On the location of zeroes of oscillatory solutions of

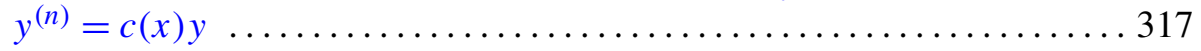

Kei Ji Izuchi, Zero sets of interpolating Blaschke products .............337

Mahesh Nerurkar, Ergodic continuous skew product actions of amenable

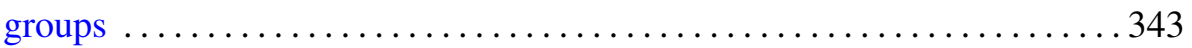

R. Owens, A maximal function characterization of a class of Hardy

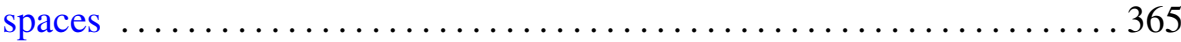

Judith Anne Packer, Point spectrum of ergodic abelian group actions and the corresponding group-measure factors $\ldots \ldots \ldots \ldots \ldots \ldots \ldots \ldots \ldots \ldots \ldots \ldots$

Judith Anne Packer, On the embedding of subalgebras corresponding to quotient actions in group-measure factors $\ldots \ldots \ldots \ldots \ldots \ldots \ldots \ldots . \ldots 4$

Iain Raeburn and Joseph L. Taylor, The bigger Brauer group and étale cohomology

David Rosen, The Diophantine equation $a x+b y=c$ in $Q(\sqrt{5})$ and other number fields

Mau-Hsiang Shih and Kok Keong Tan, Noncompact sets with convex sections

Lee Barlow Whitt, Codimension two isometric immersions between Euclidean spaces

Rodney Ian Yager, Iwasawa theory for the anticyclotomic extension 